\title{
Vector and Tensor Contributions to the Luminosity Distance
}

\author{
Enea Di Dio and Ruth Durrer \\ Département de Physique Théorique and Center for Astroparticle Physics, Université de Genève \\ 24 quai Ernest Ansermet, CH-1211 Genève 4, Switzerland
}

(Dated: November 6, 2018)

\begin{abstract}
We compute the vector and tensor contributions to the luminosity distance fluctuations in first order perturbation theory, and we expand them in spherical harmonics. This work presents the formalism with a first application to a stochastic background of primordial gravitational waves.
\end{abstract}

PACS numbers: 98.80.-k, 98.80.Es, 98.80.Jk

\section{INTRODUCTION}

The distance-redshift relation for far away objects plays an important role in cosmology. It has led Hubble, or rather Lemaître [1], to discover the expansion of the Universe; and the distance-redshift relation to far away Supernovae type Ia is at the origin of last year's Nobel Prize in physics for the discovery of the accelerated expansion of the Universe [2, 3].

A next step that has been initiated recently considers the angular and redshift fluctuations of the luminosity distance, which may also contain important information about our Universe [4 7]. One important unsolved problem is the question how strongly the distance-redshift relation may be affected by the fact that the actual Universe is not homogeneous and isotropic, but the matter distribution and also the geometry have fluctuations. To first order in perturbation theory these fluctuations can average out in the mean and are therefore expected to be small.

However, it has been found that they are significantly larger than the naively expected value that would be of the order of the gravitational potential, namely, $\sim 10^{-5}$. An analysis in first order gave fluctuations of the order of $10^{-3}$, hence 100 times larger than the naive estimate [5]. Recently, Ben-Dayan et al. [8] have calculated a second order contribution to the distance-redshift relation of the order of $\sim 10^{-3}$. Evidently, if the second order term is as large as the first order, this means that perturbation theory cannot be trusted. On the other hand, fully nonlinear toy models, which have been studied in the past, always gave relatively small modifications of the luminosity distance if the size of the fluctuations, spherical voids [9] or parallel walls [10], is small compared to the Hubble scale. Hence the problem remains open.

So far, the perturbative analyses of the distanceredshift relation have concentrated on scalar perturbations. In this work, we want to study the contributions from vector and tensor perturbations on a FriedmannLemaître (FL) universe. This is interesting for several reasons. First of all, tensor perturbations are generically produced during inflation, and hence their contribution has to be added for completeness. Second, a passing gravitational wave from some arbitrary source does generate a tensor perturbation in the distance-redshift relation to any far away object and could, at least in principle, be detected in this way. For single binary sources we have found that this effect is very small [11]; however, a stochastic background might lead to a detectable effect. Even though vector perturbations are usually not generated during inflation (and if they are they decay during the subsequent radiation dominated phase), they are relevant in many models with sources like, e.g., cosmic strings or primordial magnetic fields. A third important motivation to study vector and tensor contributions comes from the fact that at second order in perturbation theory, scalars also generate vector and tensor perturbations [12, 13]. In a complete second order treatment these have to be included. With the formalism developed in this work, such an inclusion is straight forward. We plan to report on the result of these second order contributions in a forthcoming paper [14]. A similar program is carried out in Refs. [15, 16]. There the authors discuss scalar, vector, and tensor perturbations and split them into $E$ and $B$ modes. The treatment of these papers is, however, more adapted to describe distortions of surveys and weak lensing, but the convergence calculated there is related to our distance fluctuations.

The paper is organized as follows. In the next section we discuss the luminosity-redshift relation perturbatively at first order. In Sec. III we apply these results to tensor perturbations. We first derive the general first order expressions, which we then expand in spherical harmonics. We also give a numerical example for the gravitational wave background from inflation. In Sec. IV we treat vector perturbations and in Sec. $\nabla$ we conclude. Some lengthy calculations and some details are deferred to four Appendices.

Notation: We use the metric signature $(-,+,+,+)$. We denote the derivative w.r.t. the conformal time $\eta$ with a dot.

\section{THE DISTANCE-REDSHIFT RELATION}

For an arbitrary geometry, defined through the metric $g$, a distance measure $D$ from a source moving with 4velocity $u_{S}=u\left(x_{S}\right)$ and an observer moving with 4velocity $u_{O}=u\left(x_{O}\right)$ can be obtained as a solution of the 
Sachs focusing equation [17]:

$$
\frac{d^{2} D}{d \lambda^{2}}=-\left(\mathcal{R}+|\Sigma|^{2}\right) D
$$

Here $\lambda$ is the affine parameter of a lightlike geodesic $x^{\mu}(\lambda)$ from the source to the observer, $x^{\mu}\left(\lambda_{S}\right)=x_{S}^{\mu}, x^{\mu}\left(\lambda_{O}\right)=$ $x_{O}^{\mu}$, and

$$
\mathcal{R}=\frac{1}{2} R_{\mu \nu} k^{\mu} k^{\nu} \quad \text { with } \quad k^{\mu}=\frac{d x^{\mu}}{d \lambda},
$$

$k^{\mu}$ is the 4-velocity of the lightlike geodesic, and $\Sigma$ is the complex shear of the 'screen' defined below. The source and observer are made out of baryons; hence we identify the 4 -velocity field $u^{\mu}(x)$ with the (baryonic and dark) matter velocity field.

Considering a thin light bundle with vertex at the source, the luminosity distance is given by

$$
D_{L}=(1+z) D,
$$

where $z$ denotes the source redshift, defined by

$$
1+z=\frac{\left.g_{\mu \nu} k^{\mu} u^{\nu}\right|_{S}}{\left.g_{\mu \nu} k^{\mu} u^{\nu}\right|_{O}}=\frac{\omega_{S}}{\omega_{O}} .
$$

We are considering past light cones without caustics between the observer and source positions. This is well justified as we are treating small perturbations on a Friedmann background. See Ref. [18] for more details on the effect of caustics in the past light cone.

The complex shear of the light ray bundle, $\Sigma$, is defined as follows 19]: Consider two spatial orthonormal vectors $e_{1}$ and $e_{2}$, which are normal to both the 4 -velocity $u_{O}$ and $k$ at the observer position and which are parallel transported along $k$, such that $\nabla_{k} e_{a}=0$ for $a=1,2$. The vectors $e_{1}, e_{2}$ are a basis of the so-called screen. Note that we do not require that $u$ be parallel transported along $k$; hence $e_{1}, e_{2}$ are in general not normal to $u$ elsewhere than at the observer. The complex shear is defined by

$$
\Sigma=\frac{1}{2} g\left(\epsilon, \nabla_{\epsilon} k\right)=-\frac{1}{2} g\left(\nabla_{\epsilon} \epsilon, k\right), \quad \text { with } \quad \epsilon=e_{1}+i e_{2} .
$$

We consider a light bundle with vertex at the source ${ }^{1}$. This leads to the following initial conditions (more details are found in Appendix A for the Sachs focusing equation (1)

$$
D\left(\lambda_{S}\right)=0, \quad D^{\prime}\left(\lambda_{S}\right)=\omega_{S}=-\left.g_{\mu \nu} k^{\mu} u^{\mu}\right|_{S} .
$$

In a perturbed FL metric the Sachs focusing equation (11) reduces, at first order, to

$$
\frac{d^{2} D}{d \lambda^{2}}=-\mathcal{R} D
$$

\footnotetext{
1 A light bundle with vertex at the observer yields the angular diameter distance, which is related by a factor $(1+z)^{2}$ to the luminosity distance which we determine here.
}

Since the complex scalar shear $\Sigma$ vanishes for a conformally flat spacetime, $|\Sigma|^{2}$ contributes only at second order. To first order in $\mathcal{R}$, Eq. (7) with initial conditions (6) is solved by

$$
\begin{aligned}
\frac{D\left(\lambda_{O}\right)}{\omega_{S}} & =\left(\lambda_{O}-\lambda_{S}\right)-\int_{\lambda_{S}}^{\lambda_{O}} d \lambda \int_{\lambda_{S}}^{\lambda} d \lambda^{\prime} \mathcal{R}\left(\lambda-\lambda_{S}\right) \\
& =\left(\lambda_{O}-\lambda_{S}\right)-\int_{\lambda_{S}}^{\lambda_{O}} d \lambda\left(\lambda-\lambda_{S}\right)\left(\lambda_{O}-\lambda\right) \mathcal{R},
\end{aligned}
$$

where we have used the identity

$$
\int_{\eta_{S}}^{\eta_{O}} d \eta \int_{\eta_{S}}^{\eta} d \eta^{\prime} f\left(\eta^{\prime}\right)=\int_{\eta_{S}}^{\eta_{O}} d \eta\left(\eta_{O}-\eta\right) f(\eta)
$$

for the second equal sign.

Of course, in a perturbed FL universe $\mathcal{R}$ is not first order; it also has a zeroth order contribution. But a perturbed FL universe is conformally related by the scale factor $a$ to a perturbed Minkowski spacetime and lightlike geodesics are invariant under conformal transformations. Two conformally related metrics,

$$
\tilde{g}_{\mu \nu}=a^{2} g_{\mu \nu}
$$

have the same lightlike geodesic curves, and only the affine parameter changes, $d \tilde{\lambda}=a^{2} d \lambda$, such that $\tilde{k}^{\mu}=$ $a^{-2} k^{\mu}$. Also the (normalized) matter 4 -velocity changes, $\tilde{u}^{\mu}=a^{-1} u^{\mu}$ so that the redshifts are related by

$$
\tilde{z}+1=\frac{a_{O}}{a_{S}}(\delta z+1), \quad \text { where } \frac{a_{O}}{a_{S}} \equiv \bar{z}+1
$$

is the background redshift, i.e., the redshift in an unperturbed Friedmann-Lemaitre universe, and $\delta z$ is the source redshift according to definition (4) w.r.t. to the perturbed Minkowski metric $g$, while $\tilde{z}$ is the one w.r.t. to the perturbed FL metric $\tilde{g}$. We remark that $\tilde{z}$ is the true (observed) redshift. In what follows we shall normalize the scale factor at the observer to one, $a_{O}=1$. The distance $D$ is not affected by a conformal factor, so that the effect of the expansion on the distance simply leads to a rescaling [5]

$$
\tilde{D}_{L}=(1+\bar{z}) D_{L} .
$$

We now compute the luminosity distance in a perturbed Minkowski spacetime, $D_{L}$, and then relate it to the one in a FL spacetime, $\tilde{D}_{L}$, by the above rescaling. Let $\left(1, n^{i}\right)$ be the 0 -order term of the lightlike velocity vector $k^{\mu}$ (in the nonexpanding Minkowski spacetime). The lightlike condition implies $|\mathbf{n}|^{2}=1$. We normalize the affine parameter $\lambda$ such that $\omega_{S}=k_{S}^{0}=1$ at 0th order. To determine the redshift $\delta z$, we have to solve the perturbed geodesic equation for $\mu=0$ only (in order to determine $k_{O}^{0}$ to first order), since the peculiar velocities are already first order. The Christoffel symbols of Minkowski space vanish, so that the geodesic equation for $\mu=0$ to first order is simply

$$
\frac{d k^{0}}{d \lambda}+\Gamma_{00}^{0}+2 \Gamma_{i 0}^{0} n^{i}+\Gamma_{i j}^{0} n^{i} n^{j}=0 .
$$


We normalize the affine parameter $\lambda$ such that $k_{S}^{0}=1$, and Eq. (12) is solved by

$$
k_{O}^{0}=1-\int_{\lambda_{S}}^{\lambda_{O}} d \lambda\left(\Gamma_{00}^{0}+2 \Gamma_{i 0}^{0} n^{i}+\Gamma_{i j}^{0} n^{i} n^{j}\right) .
$$

The geodesic equation (12) will be useful also in order to express the distance $D$ in terms of the conformal time $\eta$ instead of the affine parameter $\lambda$. For this we use

$$
k^{0}=\frac{d \eta}{d \lambda}=1-\int_{\lambda_{S}}^{\lambda} d \lambda^{\prime}\left(\Gamma_{00}^{0}+2 \Gamma_{i 0}^{0} n^{i}+\Gamma_{i j}^{0} n^{i} n^{j}\right),
$$

which, in first order, leads to

$$
\lambda_{O}-\lambda_{S}=\eta_{O}-\eta_{S}+\int_{\eta_{S}}^{\eta_{O}} d \eta \int_{\eta_{S}}^{\eta} d \eta^{\prime}\left(\Gamma_{00}^{0}+2 \Gamma_{i 0}^{0} n^{i}+\Gamma_{i j}^{0} n^{i} n^{j}\right) .
$$

The conformal time and the background redshift are not observable. We want to write the distance as a function of the true (observed) redshift $\tilde{z}=\bar{z}+\delta \tilde{z}$, where $\delta \tilde{z} \equiv(1+\bar{z}) \delta z$ according to Eq. (10). Following the approach presented in [5] we compute

$$
\begin{aligned}
\tilde{D}_{L}\left(\eta_{S}, \mathbf{n}\right) & =\tilde{D}_{L}(\eta(\bar{z}), \mathbf{n}) \equiv \tilde{D}_{L}(\bar{z}, \mathbf{n}) \\
& =\tilde{D}_{L}(\tilde{z}, \mathbf{n})-\left.\frac{d}{d \tilde{z}} \tilde{D}_{L}(\tilde{z}, \mathbf{n})\right|_{\tilde{z}=\bar{z}} \delta \tilde{z},
\end{aligned}
$$

with

$$
\begin{aligned}
& \begin{aligned}
\left.\frac{d}{d \tilde{z}} \tilde{D}_{L}(\tilde{z}, \mathbf{n})\right|_{\tilde{z}=\bar{z}} & =\frac{d}{d \bar{z}} \tilde{D}_{L}(\bar{z}, \mathbf{n})+\text { first order } \\
& =(1+\tilde{z})^{-1} \tilde{D}_{L}+\mathcal{H}_{S}^{-1}+\text { first order, }
\end{aligned} \\
& \text { where } \quad \mathcal{H}_{S}=\left.\frac{\dot{a}}{a}\right|_{S}
\end{aligned}
$$

In other words, we evaluate the distance at the true (observed) redshift $\tilde{D}_{L}(\tilde{z}, \mathbf{n})$ by using Eqs. (16 17) in order to relate $\tilde{D}_{L}(\tilde{z}, \mathbf{n})$ to $\tilde{D}_{L}\left(\eta_{S}, \mathbf{n}\right)$.

From Sec. III on, to simplify the notation, we denote the true (observed) redshift with $z$ instead of $\tilde{z}$. We shall not use $\tilde{z}$ anymore.

\section{THE DISTANCE-REDSHIFT RELATION FROM TENSOR PERTURBATIONS}

We first consider a perturbed Minkowski metric with tensor perturbations only, defined by

$$
d s^{2}=-d \eta^{2}+\left(\delta_{i j}+2 H_{i j}\right) d x^{i} d x^{j},
$$

where the tensor perturbations are divergence-free $H_{j, i}^{i}=0$, traceless $H_{i}^{i}=0$, symmetric $H_{i j}=H_{j i}$, and spatial $H_{\mu 0}=0$. By definition, a spin-2 perturbation is gauge-invariant. To use a notation consistent with the next section, we introduce the gauge invariant shear on the $\{t=$ constant $\}$ hypersurfaces $\sigma_{i j}=\dot{H}_{i j}$ (see, e.g., 20]).

\section{A. The perturbation equations}

From the Ricci tensor calculated in Appendix B we obtain

$$
\mathcal{R}=-\frac{1}{2} n^{i} n^{j} \square H_{i j}, \quad \text { where } \quad \square=\partial^{\mu} \partial_{\mu} .
$$

Note that this is the Minkowski space d'Alembertian, without expansion. The geodesic equation (13) for $\mu=0$ leads to (see Appendix B for details)

$$
k_{O}^{0}=1-\int_{\lambda_{S}}^{\lambda_{O}} d \lambda \sigma_{i j} n^{i} n^{j} .
$$

We consider the 4 -velocity $\left(u^{\mu}\right)=(1,0)$ because the spin2 perturbations can not source peculiar velocities at linear order, so that we obtain the redshift to first order

$$
1+\delta z=\frac{1}{k_{O}^{0}}=1+\int_{\lambda_{S}}^{\lambda_{O}} d \lambda \sigma_{i j} n^{i} n^{j} .
$$

With Eqs. 8, 11, 15) we find the luminosity distance in a perturbed FL universe with $d \tilde{s}^{2}=a^{2} d s^{2}$, as a function of the background redshift

$$
\begin{aligned}
\tilde{D}_{L}(\bar{z}, \mathbf{n}) & =(1+\bar{z})\left(\eta_{O}-\eta_{S}\right) \\
\times(1 & +\int_{\eta_{S}}^{\eta_{O}} d \eta \sigma_{i j} n^{i} n^{j} \\
& +\int_{\eta_{S}}^{\eta_{O}} d \eta \frac{\eta_{O}-\eta}{\eta_{O}-\eta_{S}} \sigma_{i j} n^{i} n^{j} \\
& \left.-\int_{\eta_{S}}^{\eta_{O}} d \eta \frac{\left(\eta-\eta_{S}\right)\left(\eta_{O}-\eta\right)}{\eta_{O}-\eta_{S}} \mathcal{R}\right) .
\end{aligned}
$$

We have again used (9) to reduce the double integral. We finally express the luminosity distance in terms of the true, observed redshift z. Using Eqs. (1617), we obtain

$$
\begin{aligned}
\tilde{D}_{L}(z, \mathbf{n}) & =(1+z)\left(\eta_{O}-\eta_{S}\right) \\
\times(1 & -\frac{\mathcal{H}_{S}^{-1}}{\eta_{O}-\eta_{S}} \int_{\eta_{S}}^{\eta_{O}} d \eta \sigma_{i j} n^{i} n^{j} \\
& +\int_{\eta_{S}}^{\eta_{O}} d \eta \frac{\eta_{O}-\eta}{\eta_{O}-\eta_{S}} \sigma_{i j} n^{i} n^{j} \\
& \left.-\int_{\eta_{S}}^{\eta_{O}} d \eta \frac{\left(\eta-\eta_{S}\right)\left(\eta_{O}-\eta\right)}{\eta_{O}-\eta_{S}} \mathcal{R}\right) .
\end{aligned}
$$

The origin of the different terms in the redshift-distance relation is as follows: the first line is the unperturbed expression for the luminosity distance in a FL universe at the observed redshift $z$, the term on the second line derives from the redshift correction, the one on the third line from the relation between the conformal time $\eta$ and the affine parameter $\lambda$, and the one on the last line from the Sachs focusing equation. We can interpret this last term as a lensing effect. The first two terms come from the perturbation of the redshift. 
For a fluid with a vanishing anisotropic stress the redshift-distance relation becomes

$$
\begin{aligned}
& \tilde{D}_{L}(z, \mathbf{n})=(1+z)\left(\eta_{O}-\eta_{S}\right) \\
& \times\left(1-\frac{\mathcal{H}_{S}^{-1}}{\eta_{O}-\eta_{S}} \int_{\eta_{S}}^{\eta_{O}} d \eta \sigma_{i j} n^{i} n^{j}\right. \\
& \quad+\int_{\eta_{S}}^{\eta_{O}} d \eta \frac{\eta_{O}-\eta}{\eta_{O}-\eta_{S}} \sigma_{i j} n^{i} n^{j} \\
& \left.\quad+\int_{\eta_{S}}^{\eta_{O}} d \eta \frac{\left(\eta-\eta_{S}\right)\left(\eta_{O}-\eta\right)}{\eta_{O}-\eta_{S}} \mathcal{H} n^{i} n^{j} \sigma_{i j}\right),
\end{aligned}
$$

where we used the Einstein equation [20]

$$
\ddot{H}_{i j}+2 \mathcal{H} \dot{H}_{i j}-\nabla^{2} H_{i j}=0,
$$

and Eq. (19) to replace $\mathcal{R}$. If the cosmic fluid is not ideal, but has anisotropic stresses, these add to the right-hand side of Eq. (25) (see [20]) and correspondingly to the last line in Eq. (24), the lensing term.

\section{B. Spherical harmonic analysis}

We want to determine the power spectrum of the luminosity distance. In the unperturbed FL background the luminosity distance to the redshift $z$ is given by

$$
\bar{D}_{L}(z)=(1+z)\left(\eta_{O}-\eta_{S}\right) .
$$

We define the relative difference in the luminosity distance as

$$
\begin{aligned}
\Delta_{L}(z, \mathbf{n})= & \frac{\tilde{D}_{L}(z, \mathbf{n})-\bar{D}_{L}(z)}{\bar{D}_{L}(z)} \\
= & \frac{1}{\eta_{O}-\eta_{S}} \int_{\eta_{S}}^{\eta_{O}} d \eta\left[-\mathcal{H}_{S}^{-1}+\left(\eta_{O}-\eta\right)+\right. \\
& \left.+\left(\eta-\eta_{S}\right)\left(\eta_{O}-\eta\right) \mathcal{H}\right] \sigma_{i j} n^{i} n^{j}
\end{aligned}
$$

Note that we evaluate the unperturbed distance at the true, observable redshift.

We are interested in the angular power spectrum of this observable, $c_{\ell}\left(z, z^{\prime}\right)$, which depends on the redshift of the two sources and is defined by the two point correlation function

$$
\begin{aligned}
& \left\langle\Delta_{L}(z, \mathbf{n}) \Delta_{L}\left(z^{\prime}, \mathbf{n}^{\prime}\right)\right\rangle \\
& \quad=\frac{1}{4 \pi} \sum_{\ell}(2 \ell+1) c_{\ell}\left(z, z^{\prime}\right) P_{\ell}\left(\mathbf{n} \cdot \mathbf{n}^{\prime}\right) .
\end{aligned}
$$

In the distance-redshift relation (23) [and, in particular, in Eq. (24) for an ideal fluid] we have several times the term $n^{i} n^{j} \sigma_{i j}(\eta, \mathbf{x}(\eta))$ where $\mathbf{x}(\eta)=\mathbf{x}_{O}-\mathbf{n}\left(\eta_{O}-\eta\right)$. In terms of its Fourier transform this is

$$
n^{i} n^{j} \sigma_{i j}(\eta, \mathbf{x}(\eta))=\int \frac{d^{3} k}{(2 \pi)^{3}} \hat{\sigma}_{i j}(\eta, \mathbf{k}) n^{i} n^{j} e^{-i \mathbf{k} \cdot \mathbf{x}(\eta)} .
$$

Without loss of generality we choose $\mathbf{x}_{O}=\mathbf{0}$. Setting

$$
\mathbf{k}=\hat{\mathbf{k}}|\mathbf{k}|=\hat{\mathbf{k}} k, \quad \mu=\hat{\mathbf{k}} \cdot \mathbf{n}, \quad \Delta \eta=\eta_{O}-\eta,
$$

we obtain

$$
n^{i} n^{j} \sigma_{i j}(\eta, \mathbf{x}(\eta))=\int \frac{d^{3} k}{(2 \pi)^{3}} \hat{\sigma}_{i j}(\eta, \mathbf{k}) n^{i} n^{j} e^{i \mu k \Delta \eta} .
$$

Writing the exponential in terms of spherical Bessel functions

$$
e^{i \mu k \Delta \eta}=\sum_{l=0}^{\infty}(2 \ell+1) i^{\ell} j_{\ell}(k \Delta \eta) P_{\ell}(\mu)
$$

we find

$$
n^{i} n^{j} \sigma_{i j}=\int \frac{d^{3} k}{(2 \pi)^{3}} n^{i} n^{j} \hat{\sigma}_{i j} \sum_{\ell=0}^{\infty}(2 \ell+1) i^{\ell} j_{\ell}(k \Delta \eta) P_{\ell}(\mu) .
$$

With respect to a helicity basis in Fourier space

$$
\left\{\mathbf{e}^{(+)}, \mathbf{e}^{(-)}, \hat{\mathbf{k}}\right\}, \quad \mathbf{e}^{( \pm)}=\frac{1}{\sqrt{2}}\left(\mathbf{e}_{1} \pm i \mathbf{e}_{2}\right)
$$

such that

$$
\mathbf{e}^{( \pm)} \cdot \mathbf{n}=\sqrt{\frac{1-\mu^{2}}{2}} e^{ \pm i \phi}
$$

we have

$$
\hat{\sigma}_{i j} \mathbf{e}^{i} \otimes \mathbf{e}^{j}=\hat{\sigma}^{+} \mathbf{e}^{(+)} \otimes \mathbf{e}^{(+)}+\hat{\sigma}^{-} \mathbf{e}^{(-)} \otimes \mathbf{e}^{(-)} .
$$

We introduce the spherical harmonics with respect to some arbitrary $z$ direction given by a unit vector e as $Y_{\ell m}(\mathbf{n}, \mathbf{e})$, since we shall use them w.r.t. different $z$ axes. The addition theorem of spherical harmonics is

$$
P_{\ell}(\mu)=\frac{4 \pi}{2 \ell+1} \sum_{m} Y_{\ell m}^{*}(\hat{\mathbf{k}}, \mathbf{e}) Y_{\ell m}(\mathbf{n}, \mathbf{e}) .
$$

Using the following spherical harmonics definition

$$
Y_{2, \pm 2}(\mathbf{n}, \hat{\mathbf{k}})=\sqrt{\frac{15}{8 \pi}} \sin ^{2} \theta e^{ \pm 2 i \phi}=\sqrt{\frac{15}{2 \pi}} \frac{1-\mu^{2}}{2} e^{ \pm 2 i \phi}
$$

we can rewrite Eq. (33) as

$$
\begin{array}{r}
n^{i} n^{j} \sigma_{i j}=\int \frac{d^{3} k}{(2 \pi)^{3}}\left(\hat{\sigma}^{+} Y_{22}(\mathbf{n}, \hat{\mathbf{k}})+\hat{\sigma}^{-} Y_{2-2}(\mathbf{n}, \hat{\mathbf{k}})\right) \\
\times \sqrt{\frac{2 \pi}{15}} \sum_{\ell, m} 4 \pi i^{\ell} j_{\ell}(k \Delta \eta) Y_{\ell m}^{*}(\hat{\mathbf{k}}, \mathbf{e}) Y_{\ell m}(\mathbf{n}, \mathbf{e}) .(39)
\end{array}
$$

We now introduce the initial tensor power spectrum $P_{H}(k)$ through

$$
\begin{aligned}
& \left\langle\hat{H}^{ \pm}\left(\eta_{S}, \mathbf{k}\right) \hat{H}^{ \pm *}\left(\eta_{S^{\prime}}, \mathbf{k}^{\prime}\right)\right\rangle \\
& \quad=(2 \pi)^{3} \delta^{(3)}\left(\mathbf{k}-\mathbf{k}^{\prime}\right) P_{H}(k) T_{k}\left(\eta_{S}\right) T_{k}\left(\eta_{S^{\prime}}\right),
\end{aligned}
$$


where $T_{k}(\eta)$ is the transfer function with the initial condition $T_{k}(\eta) \rightarrow_{(k \eta \rightarrow 0)} 1$. The $\delta^{(3)}\left(\mathbf{k}-\mathbf{k}^{\prime}\right)$ is a consequence of stochastic homogeneity. For the shear we then have

$$
\begin{aligned}
& \left\langle\hat{\sigma}^{ \pm}\left(\eta_{S}, \mathbf{k}\right) \hat{\sigma}^{ \pm *}\left(\eta_{S^{\prime}}, \mathbf{k}^{\prime}\right)\right\rangle \\
& \quad=(2 \pi)^{3} \delta^{(3)}\left(\mathbf{k}-\mathbf{k}^{\prime}\right) P_{H}(k) \dot{T}_{k}\left(\eta_{S}\right) \dot{T}_{k}\left(\eta_{S^{\prime}}\right) .
\end{aligned}
$$

Next, we express the terms in the distance-redshift relation with the help of the power spectrum of the integrand,

$$
\left\langle n^{i} n^{j} \sigma_{i j} n^{\prime l} n^{\prime k} \sigma_{l k}\right\rangle=\frac{1}{4 \pi} \sum_{\ell}(2 \ell+1) \bar{c}_{\ell}\left(\eta, \eta^{\prime}\right) P_{\ell}\left(\mathbf{n} \cdot \mathbf{n}^{\prime}\right) .
$$

A lengthy but straight forward calculation yields [20, 21]

$\bar{c}_{\ell}=\frac{1}{\pi} \frac{(\ell+2) !}{(\ell-2) !} \int d k k^{2} P_{H}(k) \dot{T}_{k}(\eta) \dot{T}_{k}\left(\eta^{\prime}\right) \frac{j_{\ell}(k \Delta \eta)}{(k \Delta \eta)^{2}} \frac{j_{\ell}\left(k \Delta \eta^{\prime}\right)}{\left(k \Delta \eta^{\prime}\right)^{2}}$.

Using the Limber approximation (see Appendix D) for the time integrals in Eq. (24), and, in particular, Eqs. (D5), (D6) and (D7), we can simplify the time integrals, and we find the coefficients (under the ideal fluid assumption)

$$
\begin{aligned}
& c_{\ell}\left(z, z^{\prime}\right) \simeq \frac{1}{\pi} \frac{(\ell+2) !}{(\ell-2) !} \frac{I_{\ell}^{2}}{\ell^{4}} \frac{1}{\Delta \eta_{S}} \frac{1}{\Delta \eta_{S^{\prime}}} \\
& \times \int_{k^{*}}^{\infty} d k P_{H}(k) \dot{T}^{2}\left(\eta_{\ell, k}\right)\left(A+B \mathcal{H}\left(\eta_{\ell, k}\right)+C \mathcal{H}^{2}\left(\eta_{\ell, k}\right)\right),
\end{aligned}
$$

where we have introduced

$$
\begin{gathered}
\Delta \eta_{S}=\eta_{O}-\eta_{S}, \quad \Delta \eta_{S^{\prime}}=\eta_{O}-\eta_{S^{\prime}}, \\
\eta_{\ell, k}=\eta_{O}-\frac{\ell}{k}, \quad I_{\ell}^{2}=\frac{1.58}{\ell}, \\
k^{*}=\max \left\{\frac{\ell}{\Delta \eta_{S}}, \frac{\ell}{\Delta \eta_{S^{\prime}}}\right\},
\end{gathered}
$$

and

$$
\begin{aligned}
A= & \mathcal{H}_{S}^{-1} \mathcal{H}_{S^{\prime}}^{-1}+\frac{\ell^{2}}{k^{2}}-\frac{\ell}{k}\left(\mathcal{H}_{S}^{-1}+\mathcal{H}_{S^{\prime}}^{-1}\right), \\
B= & -\frac{\ell}{k}\left(\mathcal{H}_{S}^{-1} \Delta \eta_{S^{\prime}}+\mathcal{H}_{S^{\prime}}^{-1} \Delta \eta_{S}\right)-2 \frac{\ell^{3}}{k^{3}} \\
& +\frac{\ell^{2}}{k^{2}}\left(\Delta \eta_{S}+\Delta \eta_{S^{\prime}}+\mathcal{H}_{S}^{-1}+\mathcal{H}_{S^{\prime}}^{-1}\right), \\
C= & \frac{\ell^{2}}{k^{2}} \Delta \eta_{S} \Delta \eta_{S^{\prime}}-\frac{\ell^{3}}{k^{3}}\left(\Delta \eta_{S}+\Delta \eta_{S^{\prime}}\right)+\frac{\ell^{4}}{k^{4}} .
\end{aligned}
$$

More details can be found in Appendix D

\section{Application}

As an example, we consider a flat primordial tensor power spectrum as expected from inflation $P_{H}(k)=$ $\alpha / k^{3}$. If $r$ denotes the tensor to scalar ratio, the scalar amplitude as measured by the Wilkinson Microwave
Anisotropy Probe experiment [22] yields $\alpha \simeq r \times 10^{-9}$, such that the tensor power spectrum becomes

$$
P_{H}(k) \simeq \frac{r}{k^{3}} 10^{-9}
$$

Considering an ideal fluid, the transfer function $T_{k}(\eta)$ is the solution of the differential equation (25),

$$
\ddot{T}_{k}(\eta)+2 \mathcal{H} \dot{T}_{k}(\eta)+k^{2} T_{k}(\eta)=0,
$$

with initial condition $T_{k}\left(\eta_{\text {in }}\right)=1$ and $\dot{T}_{k}\left(\eta_{\text {in }}\right)=0$ for $k \eta_{\text {in }} \ll 1$. In a matter (or radiation) dominated universe this differential equation can be solved analytically in terms of Bessel functions. The growing (not decaying) mode is given by

$$
T_{k}(\eta)=(k \eta)^{1 / 2-q} Y_{1 / 2-q}(k \eta), \quad \text { where } a \propto \eta^{q},
$$

and $Y_{\nu}$ is the Bessel function of the second kind of order $\nu$. At late times, when the cosmological constant dominates, we cannot write the scale factor $a(\eta)$ as a power law and we have no analytic solution to (52). To determine the $c_{\ell}$ coefficients, we have solved the differential Eq. (52) numerically.

The resulting power spectrum $c_{\ell}(z, z)$ for different source redshifts is shown in Fig. 1.

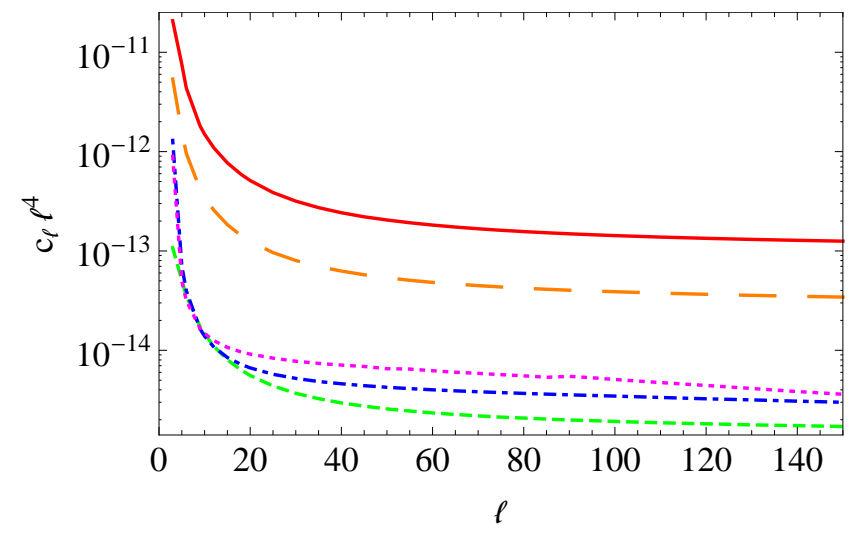

FIG. 1: We show the tensor power spectrum rescaled by $\ell^{4}$ for the fluctuations in the luminosity distance for different values of the source redshift $(z=0.5$, dotted pink line; $z=1$, dotdashed blue line; $z=2$, dashed green line; $z=3$, long-dashed orange line; $z=4$, solid red line). In the figure we have set $r=1$.

Clearly, for sufficiently large $\ell, c_{\ell}(z, z) \propto \ell^{-4}$. The simplest way to understand this scaling is to note that once a mode enters the horizon, the tensor fluctuations scale like $\int \sigma d \eta \sim H \propto a_{k} / a \propto(k \eta)^{-q}$, where $a_{k}=$ $a(\eta=1 / k)$ denotes the value of the scale factor at horizon entry. For modes that enter during the radiation era $q=1$, while for modes that enter during the matter era $q=2$. Hence $\int \sigma d \eta \propto H \propto H_{\text {in }} / k^{q}$ is acquiring a factor $k^{-q}$ with respect to the scale invariant initial spectrum. This leads to a red spectrum, $k^{3}\left(\int \sigma\right)^{2} \propto$ $k^{-2 q}$ and $\ell^{2} c_{\ell}(z, z) \propto \ell^{-2 q}$. This spectrum turns from 
$c_{\ell} \propto \ell^{-4}$ for scales that enter the horizon in the radiation era to $c_{\ell} \propto \ell^{-6}$ for scales that enter the horizon in the matter era. For $z=4$ this happens roughly at $\ell \sim 20$. Of course, the transition is quite gradual.

Comparing Fig. 1 with the results from scalar perturbations [5], we see first that the tensor contribution is much smaller, nearly 8 orders of magnitude. We obtain $\ell^{4} c_{\ell}(z) \sim 5 \times 10^{-13}$ for $z=4$ and $\ell \gtrsim 40$ while scalar perturbations yield $\ell^{2} c_{\ell}(z) \sim 10^{-5}$ for $z=4$ and $\ell \gtrsim 100$. Furthermore, despite also being proportional to the lensing term, it scales differently with $\ell$. This comes from the fact that the scalar lensing term is determined by the spectrum of $k^{2} \Psi$, where $\Psi$ is the scale invariant Bardeen potential, while for scales that enter during radiation dominated expansion, $\sigma_{i j} n^{i} n^{j}$ is suppressed by a factor of $1 / k$.

Interestingly the tensor signal is not monotonic in redshift up to $z \simeq 2$. It has a sharp minimum at $z \simeq 1.65$. To illustrate this, we also plot $c_{\ell}(z, z)$ as a function of the source redshift for different values of $\ell$ in Fig. 2

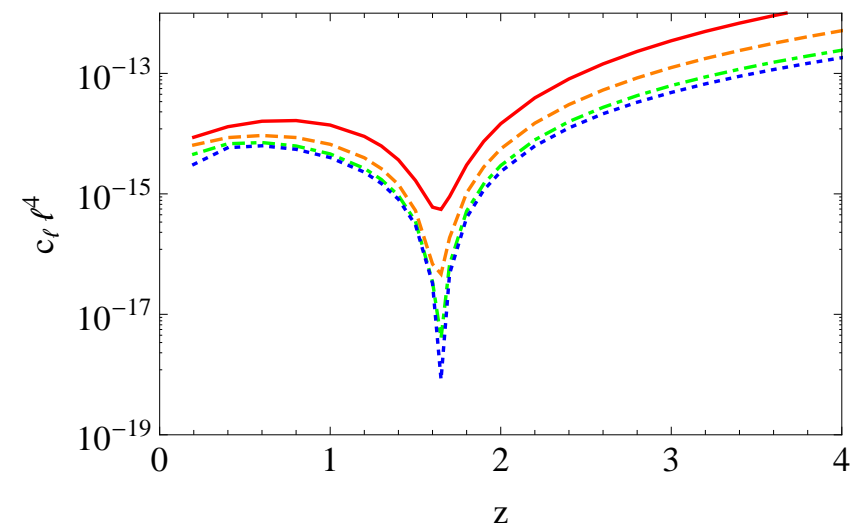

FIG. 2: We show the tensor power spectrum rescaled by $\ell^{4}$ for the fluctuations in the luminosity distance as a function of the source redshift for different values $\ell(\ell=60$, dotted blue line; $\ell=40$, dot-dashed green line; $\ell=20$, dashed orange line; $\ell=10$, solid red line). Also here $r=1$.

The signal drops to 0 at $z_{c}=1.65$. This comes from the fact that it is dominated by two terms with opposite sign. To see this, we also show the contributions from the three terms in the square bracket of (27) individually in Fig. 3 .

If the source redshift is small, $\eta_{S} \sim \eta_{O}$, the first term $\propto-\mathcal{H}_{S}^{-1} \sim-\eta_{S} / 2$ dominates, while for large redshifts, $\eta_{S} \ll \eta_{O}$, the second term $\propto\left(\eta_{O}-\eta\right)$ dominates. If $\sigma_{i j} n^{i} n^{j}$ has a definite sign, the result inherits this sign for small redshifts and the opposite sign for large redshifts. The sign changes happens around $\eta_{S}=\eta_{O} / 2$ corresponding to a redshift $z_{c} \sim 3$. This is not expected to be very precise; in particular, we have neglected the time dependence of the transfer function in this argument. The more precise numerical evaluation gives $z_{c} \simeq 1.65$. Interestingly this redshift is close to the maximum of the angular diameter distance $D_{A}(z)=\left(\eta_{O}-\eta_{S}\right) /(1+z)$.

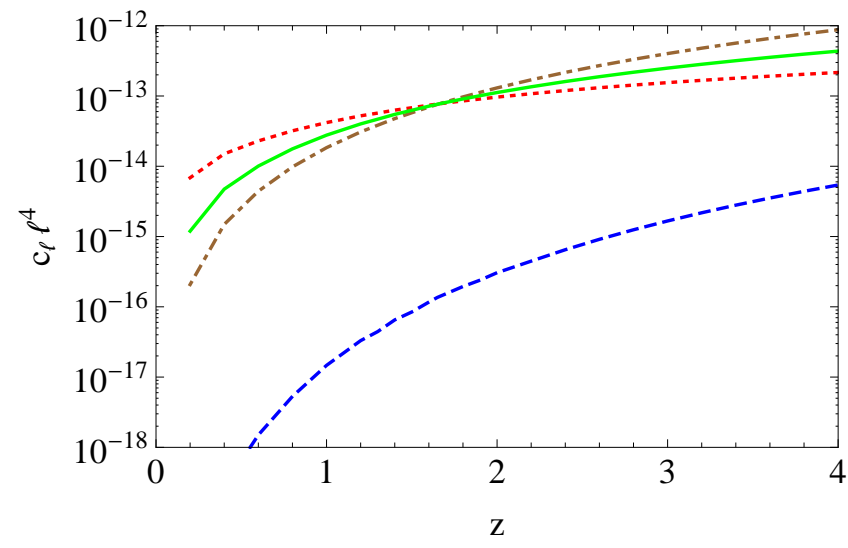

FIG. 3: We show the contributions from the different terms of Eq. (27) to the tensor power spectrum rescaled by $\ell^{4}$ for the fluctuations in the luminosity distance as a function of the source redshift for $\ell=40$. (First term: dotted red line; second term: dot-dashed brown line; third term: dashed blue line; correlator of the first and second term: solid green line). The third term is always subdominant. We plot the correlator between the first and the second term (solid line) with the opposite sign since it is negative. The second and the first terms have opposite signs, and they cross at $z_{c} \simeq 1.65$.

The results shown in Figs. 1 to 3 have been calculated with the following cosmological parameters: $h=0.7$, $H_{O}^{-1}=2997.9 h^{-1} \mathrm{Mpc}, \Omega_{m} h^{2}=0.13, \Omega_{r} h^{2}=4.17 \times$ $10^{-5}$, and $\Omega_{\Lambda}=1-\Omega_{m}-\Omega_{r}$.

\section{THE DISTANCE-REDSHIFT RELATION FROM VECTOR PERTURBATIONS}

We now consider vector perturbations. As for tensor perturbations, we can divide out the cosmic expansion for lightlike geodesics. Hence we can consider Minkowski space with purely vector perturbations. The metric is then given by

$$
d s^{2}=-d \eta^{2}-2 B_{i} d x^{i} d \eta+\left(\delta_{i j}+H_{i, j}+H_{j, i}\right) d x^{i} d x^{j},
$$

where the perturbations are divergence-free, $B_{, i}^{i}=H_{, i}^{i}=0$. Using $B_{i j}=B_{(i, j)}$ and $H_{i j}=H_{(i, j)}$, where ( ) denotes symmetrization, the shear on the constant time hypersurface is given by [20] $\sigma_{i j}=B_{i j}+\dot{H}_{i j}$ or in 3 -vector notation $\sigma_{i}=B_{i}+\dot{H}_{i}$, and $\sigma_{i j}=\sigma_{(i, j)}$. This quantity is gauge invariant [20].

\section{A. The perturbation equations}

With the Ricci tensor calculated in Appendix C1 we obtain for vector perturbations

$$
\mathcal{R}=\frac{1}{2}\left(\nabla^{2}\left(\sigma_{i} n^{i}\right)+\dot{\sigma}_{i j} n^{i} n^{j}\right) .
$$


To determine the redshift we first evaluate the geodesic solution (13) with the Christoffel symbols derived in Appendix C 1 .

$$
k_{O}^{0}=1-\int_{\lambda_{S}}^{\lambda_{O}} d \lambda \sigma_{i j} n^{i} n^{j}
$$

Vector perturbations can have a nonvanishing peculiar velocity term. We define the observer 4 -velocity $\left(u^{\mu}\right)=$ $\left(1, B^{i}+v^{i}\right)$. The peculiar velocity $v^{i}$ defined in this way is gauge invariant. It is the vorticity of the matter flow. We now obtain

$1+\delta z=\frac{-1+n_{i} v_{S}^{i}}{-k_{O}^{0}+n_{i} v_{O}^{i}}=1+n_{i}\left(v_{O}^{i}-v_{S}^{i}\right)+\int_{\lambda_{S}}^{\lambda_{O}} d \lambda \sigma_{i j} n^{i} n^{j}$

After a short calculation, using the results of Sec. III we find the luminosity distance

$$
\begin{aligned}
\tilde{D}_{L}(\bar{z}, \mathbf{n})= & (1+\bar{z})\left(\eta_{O}-\eta_{S}\right) \\
\times(1+ & n_{i} v_{O}^{i}-2 n_{i} v_{S}^{i}+\int_{\eta_{S}}^{\eta_{O}} d \eta \sigma_{i j} n^{i} n^{j} \\
& +\frac{1}{\eta_{O}-\eta_{S}} \int_{\eta_{S}}^{\eta_{O}} d \eta\left(\eta_{O}-\eta\right) \sigma_{i j} n^{i} n^{j} \\
& \left.-\int_{\eta_{S}}^{\eta_{O}} d \eta \frac{\left(\eta-\eta_{S}\right)\left(\eta_{O}-\eta\right)}{\eta_{O}-\eta_{S}} \mathcal{R}\right) .
\end{aligned}
$$

Since we are interested in expressing the luminosity distance as a function of the true redshift, we have to evaluate Eq. (58) at $z$ and subtract the correction term defined through Eqs. (16) and (17),

$$
\begin{array}{rl}
\tilde{D}_{L}(z, \mathbf{n}) & =(1+z)\left(\eta_{O}-\eta_{S}\right) \\
\times(1- & \frac{\mathcal{H}_{S}^{-1}}{\eta_{O}-\eta_{S}} n_{i} v_{O}^{i}-n_{i} v_{S}^{i}\left(1-\frac{\mathcal{H}_{S}^{-1}}{\eta_{O}-\eta_{S}}\right) \\
& -\frac{\mathcal{H}_{S}^{-1}}{\eta_{O}-\eta_{S}} \int_{\eta_{S}}^{\eta_{O}} d \eta \sigma_{i j} n^{i} n^{j} \\
& +\frac{1}{\eta_{O}-\eta_{S}} \int_{\eta_{S}}^{\eta_{O}} d \eta\left(\eta_{O}-\eta\right) \sigma_{i j} n^{i} n^{j} \\
& \left.-\int_{\eta_{S}}^{\eta_{O}} d \eta \frac{\left(\eta-\eta_{S}\right)\left(\eta_{O}-\eta\right)}{\eta_{O}-\eta_{S}} \mathcal{R}\right), \\
\Delta_{L}(z, \mathbf{n}) & =-\frac{\mathcal{H}-1}{\eta_{O}-\eta_{S}} n_{i} v_{O}^{i}-n_{i} v_{S}^{i}\left(1-\frac{\mathcal{H}_{S}^{-1}}{\eta_{O}-\eta_{S}}\right) \\
& -\frac{\mathcal{H}_{S}^{-1}}{\eta_{O}-\eta_{S}} \int_{\eta_{S}}^{\eta_{O}} d \eta \sigma_{i j} n^{i} n^{j} \\
& +\frac{1}{\eta_{O}-\eta_{S}} \int_{\eta_{S}}^{\eta_{O}} d \eta\left(\eta_{O}-\eta\right) \sigma_{i j} n^{i} n^{j} \\
& -\int_{\eta_{S}}^{\eta_{O}} d \eta\left(\eta-\eta_{S}\right)\left(\eta_{O}-\eta\right) \\
\eta_{O}-\eta_{S} & \mathcal{R} .
\end{array}
$$

This expression depends only on the gauge-invariant quantities $v^{i}$ and $\sigma_{i j}$ as it should. We note also that we did not assume any gravitational theory yet. Indeed the procedure used so far is completely geometrical. If one is interested in general relativity (GR), then the two gauge-invariant variables $v^{i}$ and $\sigma_{i j}$ are not independent but related via Einstein's equations [20],

$$
\nabla^{2} \sigma_{i}=-16 \pi G a^{2} v_{i}(\bar{\rho}+\bar{p})
$$

where $\bar{\rho}$ and $\bar{p}$ are the background density and pressure, respectively.

\section{B. Spherical harmonic analysis}

As for the tensor perturbations, we are interested in the term $n^{i} n^{j} \sigma_{i j}$. The main difference is that in the vector case we have $\sigma_{i j}=\sigma_{(i, j)}$ in real space and $\hat{\sigma}_{i j}=-i k_{(i} \hat{\sigma}_{j)}$ in Fourier space. This leads to

$$
n^{l} n^{j} \hat{\sigma}_{l j} e^{i \mu k \Delta \eta}=-k n^{j} \hat{\sigma}_{j} \frac{\partial e^{i \mu k \Delta \eta}}{\partial(k \Delta \eta)} .
$$

With this we can write Eq. (33) as

$$
n^{i} n^{j} \sigma_{i j}=-\int \frac{d^{3} k}{(2 \pi)^{3}} k n^{j} \hat{\sigma}_{j} \sum_{\ell=0}^{\infty}(2 \ell+1) i^{\ell} j_{\ell}^{\prime}(k \Delta \eta) P_{\ell}(\mu)
$$

With the helicity basis defined in Sec. III the addition theorem of the spherical harmonics (37), and

$Y_{1, \pm 1}(\mathbf{n}, \hat{\mathbf{k}})=\mp \sqrt{\frac{3}{8 \pi}} \sin \theta e^{ \pm i \phi}=\mp \sqrt{\frac{3}{4 \pi}} \sqrt{\frac{1-\mu^{2}}{2}} e^{ \pm i \phi}$,

we obtain

$$
\begin{aligned}
n^{i} n^{j} \sigma_{i j} & =\int \frac{d^{3} k}{(2 \pi)^{3}} k\left(Y_{1,1}(\mathbf{n}, \hat{\mathbf{k}}) \hat{\sigma}^{+}-Y_{1,-1}(\mathbf{n}, \hat{\mathbf{k}}) \hat{\sigma}^{-}\right) \\
& \times 4 \pi \sqrt{\frac{4 \pi}{3}} \sum_{\ell, m} i^{\ell} j_{\ell}^{\prime}(k r) Y_{\ell m}^{*}(\hat{\mathbf{k}}, \mathbf{e}) Y_{\ell m}(\mathbf{n}, \mathbf{e}) .
\end{aligned}
$$

If we assume that vector perturbations have been generated at some time in the past, we can define the vector power spectrum as for the tensor case as

$$
\begin{aligned}
& \left\langle\hat{\sigma}^{ \pm}\left(\eta_{S}, \mathbf{k}\right) \hat{\sigma}^{ \pm *}\left(\eta_{S^{\prime}}, \mathbf{k}^{\prime}\right)\right\rangle \\
& \quad=(2 \pi)^{3} \delta^{(3)}\left(\mathbf{k}-\mathbf{k}^{\prime}\right) P_{\sigma}(k) T_{k}\left(\eta_{S}\right) T_{k}\left(\eta_{S^{\prime}}\right)
\end{aligned}
$$

If we do not want to consider the case of early generation, we simply have to replace $P_{\sigma}(k) T_{k}\left(\eta_{S}\right) T_{k}\left(\eta_{S^{\prime}}\right)$ by a timedependent power spectrum, $P_{\sigma}\left(k, \eta_{S}, \eta_{S^{\prime}}\right)$. The model under consideration (e.g., cosmic strings) then has to be used to determine this time-dependent power spectrum. If, however, vector perturbations evolve freely, we can then compute the shear power spectrum as for tensors,

$$
\left\langle n^{i} n^{j} \sigma_{i j} n^{\prime l} n^{\prime k} \sigma_{l k}\right\rangle=\frac{1}{4 \pi} \sum_{\ell}(2 \ell+1) \bar{c}_{\ell}\left(\eta, \eta^{\prime}\right) P_{\ell}\left(\mathbf{n} \cdot \mathbf{n}^{\prime}\right)
$$


finding (see Appendix C2)

$$
\begin{aligned}
\bar{c}_{\ell} & =\frac{2 \ell(\ell+1)}{\pi(2 \ell+1)^{2}} \int d k k^{4} P_{\sigma}(k) T_{k}(\eta) T_{k}\left(\eta^{\prime}\right) \\
& \times\left[j_{\ell-1}^{\prime}(k r) j_{\ell-1}^{\prime}\left(k r^{\prime}\right)+j_{\ell+1}^{\prime}(k r) j_{\ell+1}^{\prime}\left(k r^{\prime}\right)\right]
\end{aligned}
$$

As mentioned above, in more realistic scenarios, where vector perturbations are generated, e.g., via anisotropic stresses from topological defects or by second order perturbations, we obtain a shear power spectrum of the form $P_{\sigma}\left(k, \eta, \eta^{\prime}\right)$, which cannot be factorized into a random initial spectrum and a deterministic transfer function.

In Appendix $\mathrm{C} 2$ we nevertheless, for sake of completeness, continue with expression (68) to derive the vector angular power spectrum for the luminosity distance fluctuations. We do not repeat the lengthy, complicated, and not very illuminating formulas here.

\section{CONCLUSIONS AND OUTLOOK}

In this paper we have calculated the angular power spectrum of the linear vector and tensor fluctuations in the distance-redshift relation. For vector perturbations we have simply derived the formulas and for tensor fluctuations we have applied them to an initial spectrum of fluctuations from inflation. It is interesting to see that the tensor-distance fluctuation spectrum is not simply suppressed by a factor $r$ as one might naively expect, but by about 8 orders of magnitude more. The reason for this is mainly that tensor fluctuations decay once they enter the horizon, while, on the contrary, scalar perturbations start growing. We therefore expect that the tensor signal generated from scalar perturbations at second order dominates over the small first order signal. The calculations of these second order contributions are left to a future project [14].

We have also found that the tensor signal is not monotonically increasing with redshift as we would expect it from a pure lensing signal. This is due to the fact that the total signal is the sum of a redshift part, $\propto-\delta z /(z+1)$, and a lensing part. At redshift $z_{c} \simeq 1.65$, which is close to the redshift where the angular diameter distance $D_{A}=\left(\eta_{O}-\eta_{S}\right) /(1+z)$ has a maximum, these terms cancel, and at higher redshifts the redshift-term dominates.

\section{Acknowledgments}

This work is supported by the Swiss National Science Foundation.

\section{Appendix A: Sachs focusing equation}

Much of this work is based on the Sachs focusing equation [17]. It has been shown [23] that the distance can be defined as

$$
D=\sqrt{|\operatorname{det} \mathcal{D}|}
$$

where $\mathcal{D}$ is the Jacobi matrix that satisfies the differential equation

$$
\frac{d^{2} \mathcal{D}}{d \lambda^{2}}=\left(\begin{array}{cc}
-\mathcal{R}-\operatorname{Re}(F) & -\operatorname{Im}(F) \\
\operatorname{Im}(F) & -\mathcal{R}+\operatorname{Re}(F)
\end{array}\right) \mathcal{D},
$$

with $F=\frac{1}{2} R_{\alpha \mu \beta \nu} \bar{\epsilon}^{\alpha} \bar{\epsilon}^{\beta} k^{\mu} k^{\nu}$. The determinant of the Jacobi matrix $\mathcal{D}$ describes the area of the thin light beam and its square root is therefore a distance, (A1), if the affine parameter $\lambda$ is normalized such that $\omega_{S}=1$. In general one can use the Sachs focusing equation also with a different affine parameter normalization. Indeed from the distance definition

$$
D=\sqrt{\frac{d A_{O}}{d \Omega_{S}}}
$$

and the solid angle aberration 17

$$
\frac{d \tilde{\Omega}}{d \Omega}=\left(\frac{k_{\mu} u^{\mu}}{k_{\mu} \tilde{u}^{\mu}}\right)^{2}=\frac{\omega^{2}}{\tilde{\omega}^{2}},
$$

we find, setting $\omega=1$,

$$
\tilde{D}=\sqrt{\frac{d A_{O}}{d \tilde{\Omega}_{S}}}=\tilde{\omega} \sqrt{\frac{d A_{O}}{d \Omega_{S}}}=\tilde{\omega} D .
$$

Since we are considering a light beam with a vertex at the source position, the initial conditions of the Sachs focusing equation are

$$
D\left(\lambda_{S}\right)=0,\left.\quad \frac{d D(\lambda)}{d \lambda}\right|_{\lambda=\lambda_{S}}=1,
$$

if we normalize $\lambda$ such that $\omega_{S}=1$. The general initial conditions for an arbitrary affine parameter $\lambda$, are given by (6). Choosing $\omega_{S}=1+z$ one obtains the luminosity distance while $\omega_{S}=(1+z)^{-1}$ gives the angular diameter distance.

\section{Appendix B: Details for tensor perturbations}

Here we write down the nonvanishing Christoffel symbols and the Ricci tensor for the metric (18),

$$
\begin{aligned}
\Gamma_{i j}^{0}= & \eta^{0 l}\left(H_{i l, j}+H_{l j, i}-H_{i j, l}\right)=\dot{H}_{i j}, \\
& \Rightarrow \sum_{i} \Gamma_{i i}^{0}=0, \\
\Gamma_{j 0}^{i}= & \eta^{i k}\left(H_{k 0, j}+H_{j k, 0}-H_{j 0, k}\right)=\dot{H}_{j i}, \\
& \Rightarrow \Gamma_{i 0}^{i}=0, \\
\Gamma_{j l}^{i}= & \eta^{i k}\left(H_{j k, l}+H_{k l, j}-H_{j l, k}\right) \\
= & H_{j i, l}+H_{i l, j}-H_{j l, i}, \\
\Gamma_{i j}^{i}= & H_{i j, i}+H_{i i, j}-H_{i j, i}=H_{i i, j}, \\
& \Rightarrow \quad \Gamma_{i j}^{i}=0 .
\end{aligned}
$$




$$
\begin{aligned}
R_{00} & =R_{i 0}=0, \\
R_{i j} & =\ddot{H}_{i j}-H_{i j, l l}=-\square H_{i j} .
\end{aligned}
$$

These components lead to

$$
\mathcal{R}=\frac{1}{2}\left(R_{00}+2 R_{i 0} n^{i}+R_{i j} n^{i} n^{j}\right)=-\frac{1}{2} n^{i} n^{j} \square H_{i j} .
$$

\section{Appendix C: Details for vector perturbations}

\section{Christoffel symbols and Ricci tensor}

Here we write down the nonvanishing Christoffel symbols and the Ricci tensor for the metric (54),

$$
\begin{aligned}
\Gamma_{00}^{i} & =-\dot{B}_{i}, \\
\Gamma_{j 0}^{i} & =\frac{1}{2}\left(\partial_{\eta}\left(H_{i, j}+H_{j, i}\right)-B_{i, j}+B_{j, i}\right), \\
\Gamma_{i 0}^{i} & =\dot{H}_{i, i} \quad \Rightarrow \quad \Gamma_{i 0}^{i}=0, \\
\Gamma_{i j}^{0} & =\frac{1}{2}\left(B_{j, i}+B_{i, j}+\dot{H}_{i, j}+\dot{H}_{j, i}\right)=\sigma_{i j}, \\
\Gamma_{i i}^{0} & =B_{i, i}+\dot{H}_{i, i} \quad \Rightarrow \quad \sum_{i} \Gamma_{i i}^{0}=0, \\
\Gamma_{i j}^{i} & =H_{i, i j} \quad \Rightarrow \quad \Gamma_{i j}^{i}=0, \\
\Gamma_{j k}^{i} & =H_{i, j k}, \quad \Rightarrow \quad \Gamma_{i k}^{i}=0, \\
\Gamma_{i k}^{i} & =H_{i, i k} \\
R_{00} & =0, \\
R_{i 0} & =\frac{1}{2}\left(B_{i, j j}+\dot{H}_{i, j j}\right)=\frac{1}{2} \nabla^{2} \sigma_{i}, \\
R_{i j} & =\frac{1}{2}\left(\dot{B}_{j, i}+\dot{B}_{i, j}+\ddot{H}_{i, j}+\ddot{H}_{j, i}\right)=\dot{\sigma}_{i j} .
\end{aligned}
$$

With these Ricci tensor components we can easily compute

$$
\begin{aligned}
\mathcal{R} & =\frac{1}{2}\left(R_{00}+2 R_{i 0} n^{i}+R_{i j} n^{i} n^{j}\right) \\
& =\frac{1}{2}\left(\nabla^{2} \sigma_{i} n^{i}+\dot{\sigma}_{i j} n^{i} n^{j}\right) .
\end{aligned}
$$

\section{The $c_{\ell}$ coefficients}

We first derive in detail Eq. (68). We use the relation

$$
Y_{1, \pm 1}(\mathbf{n}, \hat{\mathbf{k}})=\sqrt{\frac{4 \pi}{3}} \sum_{m^{\prime}=-1}^{1} Y_{1 m^{\prime}}(\mathbf{n}, \mathbf{e})_{\mp 1} Y_{1 m^{\prime}}^{*}(\hat{\mathbf{k}}, \mathbf{e}) .
$$

Here ${ }_{\mp 1} Y_{1 m^{\prime}}^{*}(\hat{\mathbf{k}}, \mathbf{e})$ is the vector spherical harmonic. The general addition theorem for spin weighted spherical harmonics used in Eq. (C13) above can be found, e.g., in [20].
Applying this to Eq. (65), we obtain

$$
\begin{aligned}
n^{i} n^{j} \sigma_{i j} & =\frac{(4 \pi)^{2}}{3} \sum_{\ell m} i^{\ell} \sum_{m^{\prime}=-1}^{1} Y_{1 m^{\prime}}(\mathbf{n}) Y_{\ell m}(\mathbf{n}) \\
\times \int & \frac{d^{3} k}{(2 \pi)^{3}} k j_{\ell}^{\prime}(k \Delta \eta) Y_{\ell m}^{*}(\hat{\mathbf{k}}) \\
& \left(\hat{\sigma}^{+}{ }_{-1} Y_{1 m^{\prime}}^{*}(\hat{\mathbf{k}})-\hat{\sigma}^{-}{ }_{1} Y_{1 m^{\prime}}^{*}(\hat{\mathbf{k}})\right) .
\end{aligned}
$$

Here we omit the arbitrary unit vector e in the notation, and we have introduced the helicity basis,

$$
\hat{\sigma}_{i}=\hat{\sigma}^{+} e_{i}^{(+)}+\hat{\sigma}^{-} e_{i}^{(-)}
$$

defined in Eq. (34). In the special case with $\mathbf{n}=\mathbf{e}$ we obtain

$$
\begin{aligned}
e^{i} e^{j} \sigma_{i j}= & \frac{4 \pi}{\sqrt{3}} \sum_{\ell} i^{\ell} \sqrt{2 \ell+1} \\
& \times \int \frac{d^{3} k}{(2 \pi)^{3}} k j_{\ell}^{\prime}(k \Delta \eta) Y_{\ell 0}^{*}(\hat{\mathbf{k}}) \\
& \quad\left(\hat{\sigma}^{+}{ }_{-1} Y_{10}^{*}(\hat{\mathbf{k}})-\hat{\sigma}^{-}{ }_{1} Y_{10}^{*}(\hat{\mathbf{k}})\right),
\end{aligned}
$$

where we have used

$$
Y_{\ell m}(\mathbf{e}, \mathbf{e})=\sqrt{\frac{2 \ell+1}{4 \pi}} \delta_{m 0},
$$

and Eq. (C13) for $\mathbf{n}=\mathbf{e}$ which yields,

$$
\begin{aligned}
Y_{1, \pm 1}(\mathbf{e}, \hat{\mathbf{k}}) & =\sqrt{\frac{4 \pi}{3}} \sum_{m^{\prime}=-1}^{1} Y_{1 m^{\prime}}(\mathbf{e}, \mathbf{e})_{\mp 1} Y_{1 m^{\prime}}^{*}(\hat{\mathbf{k}}, \mathbf{e}) \\
& ={ }_{\mp 1} Y_{10}^{*}(\hat{\mathbf{k}}, \mathbf{e}) .
\end{aligned}
$$

Since the two point correlation function (67) depends on the angle $\mathbf{n} \cdot \mathbf{n}^{\prime}$ only we can set $\mathbf{n}^{\prime}=\mathbf{e}$ without loss of generality. With this we find

$$
\begin{aligned}
& \left\langle n^{i} n^{j} \sigma_{i j} e^{i} e^{j} \sigma_{i j}\right\rangle \\
& =\frac{8}{3 \sqrt{3}} \sum_{\ell, m} \sum_{\tilde{\ell}} i^{\ell-\tilde{\ell}} \sqrt{2 \tilde{\ell}+1} Y_{\ell m}(\mathbf{n}) \sum_{m^{\prime}=-1}^{1} Y_{1 m^{\prime}}(\mathbf{n}) \\
& \quad \times \int d k k^{4} j_{\ell}^{\prime}(k \Delta \eta) j_{\tilde{\ell}}^{\prime}\left(k \Delta \eta^{\prime}\right) P_{\sigma}(k) T_{k}(\eta) T_{k}\left(\eta^{\prime}\right) \\
& \quad \times \int d \Omega_{\hat{\mathbf{k}}} f_{m^{\prime}}(\hat{\mathbf{k}}) Y_{\ell m}^{*}(\hat{\mathbf{k}}) Y_{\tilde{\ell} 0}(\hat{\mathbf{k}}),
\end{aligned}
$$

where we have introduced

$$
f_{m^{\prime}}(\hat{\mathbf{k}})={ }_{-1} Y_{1 m^{\prime}}^{*}(\hat{\mathbf{k}}){ }_{-1} Y_{10}(\hat{\mathbf{k}})+{ }_{1} Y_{1 m^{\prime}}^{*}(\hat{\mathbf{k}}){ }_{1} Y_{10}(\hat{\mathbf{k}}) .
$$

Since the spherical harmonics form an orthogonal basis on $S_{2}$, we can expand the product of two of them again in terms of spherical harmonics using the Clebsch-Gordan coefficients 24]. In the case of Eq. (C18) we use

$$
\begin{array}{r}
Y_{\ell m}^{*}(\hat{\mathbf{k}}) Y_{\tilde{\ell} 0}(\hat{\mathbf{k}})=\sum_{L=\left|\ell-\ell^{\prime}\right|}^{\ell+\ell^{\prime}} \sqrt{\frac{(2 \ell+1)(2 \tilde{\ell}+1)}{4 \pi(2 L+1)}} \\
\times\langle\ell, 0, \tilde{\ell}, 0 \mid L, 0\rangle\langle\ell, m, \tilde{\ell}, 0 \mid L, m\rangle Y_{L m}^{*}(\hat{\mathbf{k}}) .
\end{array}
$$


The dependence of the spherical harmonics on the azimuthal angle $\phi$,

$$
Y_{L m}^{*} \propto e^{-i m \phi} \quad \text { and } \quad{ }_{ \pm 1} Y_{1 m^{\prime}}^{*} \propto e^{-i m^{\prime} \phi}
$$

implies that the integral over angles in Eq. (C18) only contributes for $m^{\prime}=-m$. Therefore also $m \in\{-1,0,1\}$. Since $f_{m^{\prime}}(\hat{\mathbf{k}})$ contains only terms that either do not depend on $\theta$ or that are quadratic in $\sin (\theta)$ and $\cos (\theta)$ the only nonvanishing contributions are $L=0$ or $L=2$. Analogous to Eq. (C20) we write

$$
\begin{aligned}
& Y_{\ell, m}(\mathbf{n}) Y_{1,-m}(\mathbf{n})=\sum_{n} \sqrt{\frac{3(2 \ell+1)}{4 \pi(2 n+1)}} \\
& \quad \times\langle\ell, 0,1,0 \mid n, 0\rangle\langle\ell, m, 1,-m \mid n, 0\rangle Y_{n 0}(\mathbf{n}) .
\end{aligned}
$$

The addition theorem for the spherical harmonics implies

$$
P_{n}(\mathbf{n} \cdot \mathbf{e})=\sqrt{\frac{4 \pi}{2 n+1}} Y_{n 0}(\mathbf{n})
$$

Using these identities we can rewrite the correlation function (C18) as

$$
\begin{aligned}
& \left\langle n^{i} n^{j} \sigma_{i j} e^{i} e^{j} \sigma_{i j}\right\rangle=\sum_{n} \sum_{L=0,2} \sum_{\ell, \tilde{\ell}} \sum_{m=-1}^{1} \frac{i^{\ell-\tilde{\ell}}}{3 \pi^{3 / 2}} \\
& \times(2 \ell+1)(2 \tilde{\ell}+1)(2 L+1)^{-1 / 2}
\end{aligned}
$$

$$
\begin{aligned}
& \quad \times\langle\ell, 0, \tilde{\ell}, 0 \mid L, 0\rangle\langle\ell, m, \tilde{\ell}, 0 \mid L, m\rangle \\
& \quad \times\langle\ell, 0,1,0 \mid n, 0\rangle\langle\ell, m, 1,-m \mid n, 0\rangle \\
& \times \int d k k^{4} j_{\ell}^{\prime}(k \Delta \eta) j_{\tilde{\ell}}^{\prime}\left(k \Delta \eta^{\prime}\right) P_{\sigma}(k) T_{k}(\eta) T_{k}\left(\eta^{\prime}\right) B_{L m} P_{n}(\mathbf{n} \cdot \mathbf{e}),
\end{aligned}
$$

where we have introduced

$$
B_{L m}=\int d \Omega_{\hat{\mathbf{k}}} f_{-m}(\hat{\mathbf{k}}) Y_{L m}^{*}(\hat{\mathbf{k}})
$$

The nonvanishing coefficients are given by

$$
B_{00}=\frac{1}{\sqrt{\pi}}, \quad B_{2, \pm 1}=\frac{1}{2} \sqrt{\frac{3}{5 \pi}}, \quad B_{2,0}=-\frac{1}{\sqrt{5 \pi}} .
$$

Computing the sum of the Clebsch-Gordan coefficients, we find

$$
\begin{aligned}
& \left\langle n^{i} n^{j} \sigma_{i j} e^{i} e^{j} \sigma_{i j}\right\rangle=\sum_{\ell} \frac{\ell(\ell+1)}{2 \pi(2 \ell+1)} P_{\ell}(\mathbf{n} \cdot \mathbf{e}) \\
& \quad \times \int d k k^{4} P_{\sigma}(k) T_{k}(\eta) T_{k}\left(\eta^{\prime}\right) \\
& \quad \times\left[j_{\ell-1}^{\prime}(k \Delta \eta) j_{\ell-1}^{\prime}\left(k \Delta \eta^{\prime}\right)+j_{\ell+1}^{\prime}(k \Delta \eta) j_{\ell+1}^{\prime}\left(k \Delta \eta^{\prime}\right)\right] .
\end{aligned}
$$

Using this result for the $\bar{c}_{\ell}$ 's defined in Eq. (67), we obtain directly Eq. (68).

Now we compute the full $c_{\ell}$ coefficient defined in (28). We use the Limber approximation (Appendix (D) to do the time integrals $\int d \eta T_{k}(\eta) j_{\ell-1}^{\prime}\left(k\left(\eta_{O}-\eta\right)\right)$. We start with the Doppler terms, the first line of Eq. (60), which contribute to the dipole term only

$$
c_{1}^{\mathrm{D}}=\frac{4 \pi}{3} \frac{\mathcal{H}_{S}^{-1}}{\Delta \eta_{S}} \frac{\mathcal{H}_{S^{\prime}}^{-1}}{\Delta \eta_{S^{\prime}}}<\left|\vec{v}_{O}\right|^{2}>
$$

This dipole is the same as the one from the scalar analysis [5]. We cannot, of course, decide which part of the observer velocity comes from scalar perturbations and which part from vector perturbations. Since this dipole term is highly nonlinear, we neglect it in the subsequent analysis and consider only $\ell \geq 2$. We now determine the other terms. From the peculiar velocity of the source we obtain

$$
c_{\ell}^{(1)}=\frac{2 \ell(\ell+1)}{\pi(2 \ell+1)^{2}}\left(1-\frac{\mathcal{H}_{S}^{-1}}{\Delta \eta_{S}}\right)\left(1-\frac{\mathcal{H}_{S^{\prime}}^{-1}}{\Delta \eta_{S^{\prime}}}\right) \int d k k^{2} P_{v}(k) T_{k}^{v}\left(\eta_{S}\right) T_{k}^{v}\left(\eta_{S^{\prime}}\right) \sum_{\tilde{\ell}=\ell-1, \ell+1} j_{\tilde{\ell}}\left(k \Delta \eta_{S}\right) j_{\tilde{\ell}}\left(k \Delta \eta_{S^{\prime}}\right),
$$

where we have introduced the velocity power spectrum defined by

$$
\left\langle\hat{v}^{ \pm}\left(\eta_{S}, \mathbf{k}\right) \hat{v}^{ \pm *}\left(\eta_{S^{\prime}}, \mathbf{k}^{\prime}\right)\right\rangle=(2 \pi)^{3} \delta^{(3)}\left(\mathbf{k}-\mathbf{k}^{\prime}\right) P_{v}(k) T_{k}^{v}\left(\eta_{S}\right) T_{k}^{v}\left(\eta_{S^{\prime}}\right)
$$

and $\hat{v}^{ \pm}$is the peculiar velocity in terms of the helicity basis defined in Eq. (34).

The second line of the redshift-distance relation (60) leads to

$$
\begin{aligned}
c_{\ell}^{(2)} & \cong \frac{\ell(1+\ell)}{2 \pi(1+2 \ell)^{2}} \frac{\mathcal{H}_{S}^{-1}}{\Delta \eta_{S}} \frac{\mathcal{H}_{S^{\prime}}^{-1}}{\Delta \eta_{S^{\prime}}} \int d k k^{2} P_{\sigma}(k) \\
& \times \sum_{\tilde{\ell}=\ell-1, \ell+1}\left[I_{\tilde{\ell}-1}^{2} T_{k}^{2}\left(\eta_{\tilde{\ell}-1, k}\right) \Theta\left(\eta_{\tilde{\ell}-1, k}-\eta_{S}\right) \Theta\left(\eta_{\tilde{\ell}-1, k}-\eta_{S^{\prime}}\right)+\left(\frac{\tilde{\ell}+1}{\tilde{\ell}}\right)^{2} I_{\tilde{\ell}}^{2} T_{k}^{2}\left(\eta_{\tilde{\ell}, k}\right) \Theta\left(\eta_{\tilde{\ell}, k}-\eta_{S}\right) \Theta\left(\eta_{\tilde{\ell}, k}-\eta_{S^{\prime}}\right)\right.
\end{aligned}
$$




$$
\left.-\frac{\tilde{\ell}+1}{\tilde{\ell}} I_{\tilde{\ell}-1} I_{\tilde{\ell}} T_{k}\left(\eta_{\tilde{\ell}-1, k}\right) T_{k}\left(\eta_{\tilde{\ell}, k}\right)\left(\Theta\left(\eta_{\tilde{\ell}, k}-\eta_{S}\right) \Theta\left(\eta_{\tilde{\ell}-1, k}-\eta_{S^{\prime}}\right)+\Theta\left(\eta_{\tilde{\ell}-1, k}-\eta_{S}\right) \Theta\left(\eta_{\tilde{\ell}, k}-\eta_{S^{\prime}}\right)\right)\right] .(
$$

Analogously the third line yields

$$
\begin{aligned}
& c_{\ell}^{(3)} \cong \frac{\ell(1+\ell)}{2 \pi(1+2 \ell)^{2}} \frac{1}{\Delta \eta_{S}} \frac{1}{\Delta \eta_{S^{\prime}}} \int d k P_{\sigma}(k) \\
& \quad \times \sum_{\tilde{\ell}=\ell-1, \ell+1}\left[(\tilde{\ell}-1)^{2} I_{\tilde{\ell}-1}^{2} T_{k}^{2}\left(\eta_{\tilde{\ell}-1, k}\right) \Theta\left(\eta_{\tilde{\ell}-1, k}-\eta_{S}\right) \Theta\left(\eta_{\tilde{\ell}-1, k}-\eta_{S^{\prime}}\right)+(\tilde{\ell}+1)^{2} I_{\tilde{\ell}}^{2} T_{k}^{2}\left(\eta_{\tilde{\ell}, k}\right) \Theta\left(\eta_{\tilde{\ell}, k}-\eta_{S}\right) \Theta\left(\eta_{\tilde{\ell}, k}-\eta_{S^{\prime}}\right)\right. \\
&\left.\quad-\left(\tilde{\ell}^{2}-1\right) I_{\tilde{\ell}-1} I_{\tilde{\ell}} T_{k}\left(\eta_{\tilde{\ell}-1, k}\right) T_{k}\left(\eta_{\tilde{\ell}, k}\right)\left(\Theta\left(\eta_{\tilde{\ell}-1, k}-\eta_{S}\right) \Theta\left(\eta_{\tilde{\ell}, k}-\eta_{S^{\prime}}\right)+\Theta\left(\eta_{\tilde{\ell}, k}-\eta_{S}\right) \Theta\left(\eta_{\tilde{\ell}-1, k}-\eta_{S^{\prime}}\right)\right)\right] .(\mathrm{C} 32)
\end{aligned}
$$

The fourth line is composed of the two terms that contribute to $\mathcal{R}$ given in Eq. (55). Denoting them with superscripts (41), (42), and their correlation with (412) we obtain

$$
\begin{aligned}
& c_{\ell}^{(41)} \cong \frac{\ell(1+\ell)}{8 \pi(1+2 \ell)^{2}} \frac{1}{\Delta \eta_{S}} \frac{1}{\Delta \eta_{S^{\prime}}} \int d k k^{2} P_{\sigma}(k) \sum_{\tilde{\ell}=\ell-1, \ell+1}\left[\tilde{\ell}^{2}\left(\eta_{\tilde{\ell}, k}-\eta_{S}\right)\left(\eta_{\tilde{\ell}, k}-\eta_{S^{\prime}}\right) I_{\tilde{\ell}}^{2} T_{k}^{2}\left(\eta_{\tilde{\ell}, k}\right) \Theta\left(\eta_{\tilde{\ell}, k}-\eta_{S}\right) \Theta\left(\eta_{\tilde{\ell}, k}-\eta_{S^{\prime}}\right)\right], \\
& c_{\ell}^{(42)} \cong \frac{\ell(1+\ell)}{8 \pi(1+2 \ell)^{2}} \frac{1}{\Delta \eta_{S}} \frac{1}{\Delta \eta_{S^{\prime}}} \int d k P_{\sigma}(k) \\
& \times \sum_{\tilde{\ell}=\ell-1, \ell+1}\left[\left(\eta_{\tilde{\ell}-1, k}-\eta_{S}\right)\left(\eta_{\tilde{\ell}-1, k}-\eta_{S^{\prime}}\right)(\tilde{\ell}-1)^{2} I_{\tilde{\ell}-1}^{2} \dot{T}_{k}^{2}\left(\eta_{\tilde{\ell}-1, k}\right) \Theta\left(\eta_{\tilde{\ell}-1, k}-\eta_{S}\right) \Theta\left(\eta_{\tilde{\ell}-1, k}-\eta_{S^{\prime}}\right)\right. \\
& +\left(\eta_{\tilde{\ell}, k}-\eta_{S}\right)\left(\eta_{\tilde{\ell}, k}-\eta_{S^{\prime}}\right)(\tilde{\ell}+1)^{2} I_{\tilde{\ell}}^{2} \dot{T}_{k}^{2}\left(\eta_{\tilde{\ell}-1, k}\right) \Theta\left(\eta_{\tilde{\ell}, k}-\eta_{S}\right) \Theta\left(\eta_{\tilde{\ell}, k}-\eta_{S^{\prime}}\right) \\
& -\left(\tilde{\ell}^{2}-1\right) I_{\tilde{\ell}-1} I_{\tilde{\ell}} \dot{T}_{k}\left(\eta_{\tilde{\ell}-1, k}\right) \dot{T}_{k}\left(\eta_{\tilde{\ell}, k}\right)\left(\left(\eta_{\tilde{\ell}-1, k}-\eta_{S}\right)\left(\eta_{\tilde{\ell}, k}-\eta_{S^{\prime}}\right) \Theta\left(\eta_{\tilde{\ell}-1, k}-\eta_{S}\right) \Theta\left(\eta_{\tilde{\ell}, k}-\eta_{S^{\prime}}\right)\right. \\
& \left.\left.+\left(\eta_{\tilde{\ell}, k}-\eta_{S}\right)\left(\eta_{\tilde{\ell}-1, k}-\eta_{S^{\prime}}\right) \Theta\left(\eta_{\tilde{\ell}, k}-\eta_{S}\right) \Theta\left(\eta_{\tilde{\ell}-1, k}-\eta_{S^{\prime}}\right)\right)\right], \\
& c_{\ell}^{(412)} \cong \frac{\ell(1+\ell)}{8 \pi(1+2 \ell)^{2}} \frac{1}{\Delta \eta_{S}} \frac{1}{\Delta \eta_{S^{\prime}}} \int d k k P_{\sigma}(k) \\
& \times \sum_{\tilde{\ell}=\ell-1, \ell+1}\left[\left(\eta_{\tilde{\ell}, k}-\eta_{S}\right)\left(\eta_{\tilde{\ell}-1, k}-\eta_{S^{\prime}}\right) \tilde{\ell}(\tilde{\ell}-1) I_{\tilde{\ell}-1} I_{\tilde{\ell}} \dot{T}_{k}\left(\eta_{\tilde{\ell}-1, k}\right) T_{k}\left(\eta_{\tilde{\ell}, k}\right) \Theta\left(\eta_{\tilde{\ell}, k}-\eta_{S}\right) \Theta\left(\eta_{\tilde{\ell}-1, k}-\eta_{S^{\prime}}\right)\right. \\
& \left.-\left(\eta_{\tilde{\ell}, k}-\eta_{S}\right)\left(\eta_{\tilde{\ell}, k}-\eta_{S^{\prime}}\right) \tilde{\ell}(\tilde{\ell}+1) I_{\tilde{\ell}}^{2} T_{k}\left(\eta_{\tilde{\ell}, k}\right) \dot{T}_{k}\left(\eta_{\tilde{\ell}, k}\right) \Theta\left(\eta_{\tilde{\ell}, k}-\eta_{S}\right) \Theta\left(\eta_{\tilde{\ell}, k}-\eta_{S^{\prime}}\right)\right] \\
& +\left(S \leftrightarrow S^{\prime}\right)
\end{aligned}
$$

Next, we compute the cross terms between the different lines of the distance-redshift relation (59). We start with the second and third lines,

$$
\begin{aligned}
c_{\ell}^{(23)} \cong & -\frac{\ell(1+\ell)}{2 \pi(1+2 \ell)^{2}} \frac{\mathcal{H}_{S}^{-1}}{\Delta \eta_{S}} \frac{1}{\Delta \eta_{S^{\prime}}} \int d k k P_{\sigma}(k) \\
& \times \sum_{\tilde{\ell}=\ell-1, \ell+1}\left[(\tilde{\ell}-1) I_{\tilde{\ell}-1}^{2} T_{k}^{2}\left(\eta_{\tilde{\ell}-1, k}\right) \Theta\left(\eta_{\tilde{\ell}-1, k}-\eta_{S}\right) \Theta\left(\eta_{\tilde{\ell}-1, k}-\eta_{S^{\prime}}\right)+\frac{(\tilde{\ell}+1)^{2}}{\tilde{\ell}} I_{\tilde{\ell}}^{2} T_{k}^{2}\left(\eta_{\tilde{\ell}, k}\right) \Theta\left(\eta_{\tilde{\ell}, k}-\eta_{S}\right) \Theta\left(\eta_{\tilde{\ell}, k}-\eta_{S^{\prime}}\right)\right. \\
& \quad-(\tilde{\ell}+1) I_{\tilde{\ell}-1} I_{\tilde{\ell}} T_{k}\left(\eta_{\tilde{\ell}-1, k}\right) T_{k}\left(\eta_{\tilde{\ell}, k}\right) \Theta\left(\eta_{\tilde{\ell}-1, k}-\eta_{S}\right) \Theta\left(\eta_{\tilde{\ell}, k}-\eta_{S^{\prime}}\right) \\
& \left.\quad-\frac{\tilde{\ell}^{2}-1}{\tilde{\ell}} I_{\tilde{\ell}-1} I_{\tilde{\ell}} T_{k}\left(\eta_{\tilde{\ell}-1, k}\right) T_{k}\left(\eta_{\tilde{\ell}, k}\right) \Theta\left(\eta_{\tilde{\ell}, k}-\eta_{S}\right) \Theta\left(\eta_{\tilde{\ell}-1, k}-\eta_{S^{\prime}}\right)\right]+\left(S \leftrightarrow S^{\prime}\right) . \\
& (\mathrm{C} 36)
\end{aligned}
$$

The second and fourth lines yield

$$
c_{\ell}^{(241)} \cong \frac{\ell(1+\ell)}{4 \pi(1+2 \ell)^{2}} \frac{\mathcal{H}_{S}^{-1}}{\Delta \eta_{S}} \frac{1}{\Delta \eta_{S^{\prime}}} \int d k k^{2} P_{\sigma}(k)
$$




$$
\begin{aligned}
& \times \sum_{\tilde{\ell}=\ell-1, \ell+1}\left[\tilde{\ell}\left(\eta_{\ell, k}-\eta_{S^{\prime}}\right) I_{\tilde{\ell}-1} I_{\tilde{\ell}} T_{k}\left(\eta_{\tilde{\ell}-1, k}\right) T_{k}\left(\eta_{\tilde{\ell}, k}\right) \Theta\left(\eta_{\tilde{\ell}-1, k}-\eta_{S}\right) \Theta\left(\eta_{\tilde{\ell}, k}-\eta_{S^{\prime}}\right)\right. \\
& \left.-(\tilde{\ell}+1)\left(\eta_{\tilde{\ell}, k}-\eta_{S^{\prime}}\right) I_{\tilde{\ell}}^{2} T_{k}^{2}\left(\eta_{\tilde{\ell}, k}\right) \Theta\left(\eta_{\tilde{\ell}, k}-\eta_{S}\right) \Theta\left(\eta_{\tilde{\ell}, k}-\eta_{S^{\prime}}\right)\right] \quad+\left(S \leftrightarrow S^{\prime}\right), \\
& c_{\ell}^{(242)} \cong \frac{\ell(1+\ell)}{4 \pi(1+2 \ell)^{2}} \frac{\mathcal{H}_{S}^{-1}}{\Delta \eta_{S}} \frac{1}{\Delta \eta_{S^{\prime}}} \int d k k P_{\sigma}(k) \\
& \times \sum_{\tilde{\ell}=\ell-1, \ell+1}\left[\left(\eta_{\tilde{\ell}-1, k}-\eta_{S^{\prime}}\right)(\tilde{\ell}-1) I_{\tilde{\ell}-1}^{2} T_{k}\left(\eta_{\tilde{\ell}-1, k}\right) \dot{T}_{k}\left(\eta_{\tilde{\ell}-1}\right) \Theta\left(\eta_{\tilde{\ell}-1, k}-\eta_{S}\right) \Theta\left(\eta_{\tilde{\ell}-1, k}-\eta_{S^{\prime}}\right)\right. \\
& -\left(\eta_{\tilde{\ell}, k}-\eta_{S^{\prime}}\right)(\tilde{\ell}+1) I_{\tilde{\ell}-1} I_{\tilde{\ell}} T_{k}\left(\eta_{\tilde{\ell}-1, k}\right) \dot{T}_{k}\left(\eta_{\tilde{\ell}, k}\right) \Theta\left(\eta_{\tilde{\ell}-1, k}-\eta_{S}\right) \Theta\left(\eta_{\tilde{\ell}, k}-\eta_{S^{\prime}}\right) \\
& -\left(\eta_{\tilde{\ell}-1, k}-\eta_{S^{\prime}}\right) \frac{\tilde{\ell}^{2}-1}{\tilde{\ell}} I_{\tilde{\ell}-1} I_{\tilde{\ell}} \dot{T}_{k}\left(\eta_{\tilde{\ell}-1, k}\right) T_{k}\left(\eta_{\tilde{\ell}, k}\right) \Theta\left(\eta_{\tilde{\ell}, k}-\eta_{S}\right) \Theta\left(\eta_{\tilde{\ell}-1, k}-\eta_{S^{\prime}}\right) \\
& \left.+\left(\eta_{\tilde{\ell}, k}-\eta_{S^{\prime}}\right) \frac{(\tilde{\ell}+1)^{2}}{\tilde{\ell}} I_{\tilde{\ell}}^{2} T_{k}\left(\eta_{\tilde{\ell}, k}\right) \dot{T}_{k}\left(\eta_{\tilde{\ell}, k}\right) \Theta\left(\eta_{\tilde{\ell}, k}-\eta_{S}\right) \Theta\left(\eta_{\tilde{\ell}, k}-\eta_{S^{\prime}}\right)\right]+\left(S \leftrightarrow S^{\prime}\right),
\end{aligned}
$$

and, the third and fourth lines give

$$
\begin{aligned}
& c_{\ell}^{(341)} \cong-\frac{\ell(1+\ell)}{4 \pi(1+2 \ell)^{2}} \frac{1}{\Delta \eta_{S}} \frac{1}{\Delta \eta_{S^{\prime}}} \int d k k P_{\sigma}(k) \\
& \times \sum_{\tilde{\ell}=\ell-1, \ell+1}\left[\left(\eta_{\tilde{\ell}, k}-\eta_{S^{\prime}}\right) \tilde{\ell}(\tilde{\ell}-1) I_{\tilde{\ell}-1} I_{\tilde{\ell}} T_{k}\left(\eta_{\tilde{\ell}-1, k}\right) T_{k}\left(\eta_{\tilde{\ell}, k}\right) \Theta\left(\eta_{\tilde{\ell}-1, k}-\eta_{S}\right) \Theta\left(\eta_{\tilde{\ell}, k}-\eta_{S^{\prime}}\right)\right. \\
& \left.-\left(\eta_{\tilde{\ell}, k}-\eta_{S^{\prime}}\right) \tilde{\ell}(\tilde{\ell}+1) I_{\tilde{\ell}}^{2} T_{k}^{2}\left(\eta_{\tilde{\ell}, k}\right) \Theta\left(\eta_{\tilde{\ell}, k}-\eta_{S}\right) \Theta\left(\eta_{\tilde{\ell}, k}-\eta_{S^{\prime}}\right)\right] \quad+\left(S \leftrightarrow S^{\prime}\right), \\
& c_{\ell}^{(342)} \cong-\frac{\ell(1+\ell)}{4 \pi(1+2 \ell)^{2}} \frac{1}{\Delta \eta_{S}} \frac{1}{\Delta \eta_{S^{\prime}}} \int d k P_{\sigma}(k) \\
& \times \sum_{\tilde{\ell}=\ell-1, \ell+1}\left[(\tilde{\ell}-1)^{2}\left(\eta_{\tilde{\ell}-1, k}-\eta_{S^{\prime}}\right) I_{\tilde{\ell}-1}^{2} T_{k}\left(\eta_{\tilde{\ell}-1, k}\right) \dot{T}_{k}\left(\eta_{\tilde{\ell}-1, k}\right) \Theta\left(\eta_{\tilde{\ell}-1, k}-\eta_{S}\right) \Theta\left(\eta_{\tilde{\ell}-1, k}-\eta_{S^{\prime}}\right)\right. \\
& -\left(\tilde{\ell}^{2}-1\right)\left(\eta_{\tilde{\ell}, k}-\eta_{S^{\prime}}\right) I_{\tilde{\ell}-1} I_{\tilde{\ell}} T_{k}\left(\eta_{\tilde{\ell}-1, k}\right) \dot{T}_{k}\left(\eta_{\tilde{\ell}, k}\right) \Theta\left(\eta_{\tilde{\ell}-1, k}-\eta_{S}\right) \Theta\left(\eta_{\tilde{\ell}, k}-\eta_{S^{\prime}}\right) \\
& \left.-\left(\tilde{\ell}^{2}-1\right)\left(\eta_{\tilde{\ell}-1, k}-\eta_{S^{\prime}}\right) I_{\tilde{\ell}-1} I_{\tilde{\ell}} \dot{T}_{k}\left(\eta_{\tilde{\ell}-1, k}\right) T_{k}\left(\eta_{\tilde{\ell}, k}\right) \Theta\left(\eta_{\tilde{\ell}, k}-\eta_{S}\right) \Theta\left(\eta_{\tilde{\ell}-1, k}-\eta_{S^{\prime}}\right]\right) \\
& \left.+(\tilde{\ell}+1)^{2}\left(\eta_{\tilde{\ell}, k}-\eta_{S^{\prime}}\right) I_{\tilde{\ell}}^{2} T_{k}\left(\eta_{\tilde{\ell}, k}\right) \dot{T}_{k}\left(\eta_{\tilde{\ell}, k}\right) \Theta\left(\eta_{\tilde{\ell}, k}-\eta_{S}\right) \Theta\left(\eta_{\tilde{\ell}, k}-\eta_{S^{\prime}}\right)\right]+\left(S \leftrightarrow S^{\prime}\right) .
\end{aligned}
$$

To determine the correlation between the peculiar velocities and the shear on the constant time hypersurface $\sigma_{i j}$ we need to specify the gravitation theory. We choose GR by using the Einstein's equations (61). Correlating the term for the peculiar velocity of the source $v_{S}$ with the others, we find

$$
\begin{aligned}
& c_{\ell}^{(12)}=\frac{-1}{16 \pi G a_{S}^{2}\left(\bar{\rho}_{S}+\bar{p}_{s}\right)}\left(1-\frac{\mathcal{H}_{S}^{-1}}{\Delta \eta_{S}}\right) \frac{\mathcal{H}_{S^{\prime}}^{-1} 2 \ell(\ell+1)}{\Delta \eta_{S^{\prime}} \pi(2 \ell+1)^{2}} \int d k k^{5} P_{\sigma}(k) T_{k}\left(\eta_{S}\right) \sum_{\tilde{\ell}=\ell-1, \ell+1} j_{\tilde{\ell}}\left(k \Delta \eta_{S}\right) \int_{\eta_{S^{\prime}}}^{\eta_{O}} d \eta T_{k}(\eta) j_{\tilde{\ell}}^{\prime}(k \Delta \eta) \\
& +\left(S \leftrightarrow S^{\prime}\right) \\
& \cong \frac{-1}{16 \pi G a_{S}^{2}\left(\bar{\rho}_{S}+\bar{p}_{s}\right)}\left(1-\frac{\mathcal{H}_{S}^{-1}}{\Delta \eta_{S}}\right) \frac{\mathcal{H}_{S^{\prime}}^{-1} 2 \ell(\ell+1)}{\Delta \eta_{S^{\prime}} \pi(2 \ell+1)_{\tilde{\ell}=\ell-1, \ell+1}^{2}} \sum_{\frac{I_{\tilde{\ell}} \tilde{\ell}^{4}}{\Delta \eta_{S}^{5}}} P_{\sigma}\left(\frac{\tilde{\ell}}{\Delta \eta_{S}}\right) T_{\tilde{\ell} / \Delta \eta_{S}}\left(\eta_{S}\right) \\
& \left.\times\left(T_{\tilde{\ell} / \Delta \eta_{S}}\left(\eta_{S}+\frac{\Delta \eta_{S}}{\tilde{\ell}}\right) I_{\tilde{\ell}-1} \Theta\left(\eta_{S}-\eta_{S^{\prime}}+\frac{\Delta \eta_{S}}{\tilde{\ell}}\right)-T_{\tilde{\ell} / \Delta \eta_{S}}\left(\eta_{S}\right) I_{\tilde{\ell}} \frac{\tilde{\ell}+1}{\tilde{\ell}} \Theta\left(\eta_{S}-\eta_{S^{\prime}}\right)\right)\right]+\left(S \leftrightarrow S^{\prime}\right), \\
& c_{\ell}^{(13)}=\frac{1}{16 \pi G a_{S}^{2}\left(\bar{\rho}_{S}+\bar{p}_{s}\right)}\left(1-\frac{\mathcal{H}_{S}^{-1}}{\Delta \eta_{S}}\right) \frac{1}{\Delta \eta_{S^{\prime}}} \frac{2 \ell(\ell+1)}{\pi(2 \ell+1)^{2}} \int d k k^{5} P_{\sigma}(k) T_{k}\left(\eta_{S}\right) \sum_{\tilde{\ell}=\ell-1, \ell+1} j_{\tilde{\ell}}\left(k \Delta \eta_{S}\right) \int_{\eta_{S^{\prime}}}^{\eta_{O}} d \eta\left(\eta_{O}-\eta\right) T_{k}(\eta) j_{\tilde{\ell}}^{\prime}(k \Delta \eta) \\
& +\left(S \leftrightarrow S^{\prime}\right)
\end{aligned}
$$




$$
\begin{aligned}
& \cong \frac{1}{16 \pi G a_{S}^{2}\left(\bar{\rho}_{S}+\bar{p}_{s}\right)}\left(1-\frac{\mathcal{H}_{S}^{-1}}{\Delta \eta_{S}}\right) \frac{1}{\Delta \eta_{S^{\prime}} \pi(2 \ell+1)^{2}} \sum_{\tilde{\ell}=\ell-1, \ell+1}\left[\frac{I_{\tilde{\ell}} \tilde{\ell}^{3}}{\Delta \eta_{S}^{4}} P_{\sigma}\left(\frac{\tilde{\ell}}{\Delta \eta_{S}}\right) T_{\tilde{\ell} / \Delta \eta_{S}}\left(\eta_{S}\right)\right. \\
& \left.\times\left(T_{\tilde{\ell} / \Delta \eta_{S}}\left(\eta_{S}+\frac{\Delta \eta_{S}}{\tilde{\ell}}\right) I_{\tilde{\ell}-1}(\tilde{\ell}-1) \Theta\left(\eta_{S}-\eta_{S^{\prime}}+\frac{\Delta \eta_{S}}{\tilde{\ell}}\right)-T_{\tilde{\ell} / \Delta \eta_{S}}\left(\eta_{S}\right) I_{\tilde{\ell}}(\tilde{\ell}+1) \Theta\left(\eta_{S}-\eta_{S^{\prime}}\right)\right)\right]+\left(S \leftrightarrow S^{\prime}\right), \\
& c_{\ell}^{(141)}=\frac{1}{8 \pi G a_{S}^{2}\left(\bar{\rho}_{S}+\bar{p}_{s}\right)}\left(1-\frac{\mathcal{H}_{S}^{-1}}{\Delta \eta_{S}}\right) \frac{1}{\Delta \eta_{S^{\prime}}} \frac{2 \ell(\ell+1)}{\pi(2 \ell+1)^{2}} \\
& \times \int d k k^{6} P_{\sigma}(k) T_{k}\left(\eta_{S}\right) \sum_{\tilde{\ell}=\ell-1, \ell+1} j_{\tilde{\ell}}\left(k \Delta \eta_{S}\right) \int_{\eta_{S^{\prime}}}^{\eta_{O}} d \eta\left(\eta_{O}-\eta\right)\left(\eta-\eta_{S^{\prime}}\right) T_{k}(\eta) j_{\tilde{\ell}}^{\prime}(k \Delta \eta) \quad+\left(S \leftrightarrow S^{\prime}\right) \\
& \cong \frac{1}{8 \pi G a_{S}^{2}\left(\bar{\rho}_{S}+\bar{p}_{s}\right)}\left(1-\frac{\mathcal{H}_{S}^{-1}}{\Delta \eta_{S}}\right) \frac{1}{\Delta \eta_{S^{\prime}}} \frac{2 \ell(\ell+1)}{\pi(2 \ell+1)^{2}} \sum_{\tilde{\ell}=\ell-1, \ell+1}\left[\frac{I_{\tilde{\ell}} \tilde{\ell}^{4}}{\Delta \eta_{S}^{5}} P_{\sigma}\left(\frac{\tilde{\ell}}{\Delta \eta_{S}}\right) T_{\tilde{\ell} / \Delta \eta_{S}}\left(\eta_{S}\right)\right. \\
& \times\left(T_{\tilde{\ell} / \Delta \eta_{S}}\left(\eta_{S}+\frac{\Delta \eta_{S}}{\tilde{\ell}}\right) I_{\tilde{\ell}-1}(\tilde{\ell}-1)\left(\eta_{S}-\eta_{S^{\prime}}+\frac{\Delta \eta_{S}}{\tilde{\ell}}\right) \Theta\left(\eta_{S}-\eta_{S^{\prime}}+\frac{\Delta \eta_{S}}{\tilde{\ell}}\right)\right. \\
& \left.\left.-T_{\tilde{\ell} / \Delta \eta_{S}}\left(\eta_{S}\right) I_{\tilde{\ell}}(\tilde{\ell}+1)\left(\eta_{S}-\eta_{S^{\prime}}\right) \Theta\left(\eta_{S}-\eta_{S^{\prime}}\right)\right)\right]+\left(S \leftrightarrow S^{\prime}\right) \\
& c_{\ell}^{(142)}=\frac{1}{8 \pi G a_{S}^{2}\left(\bar{\rho}_{S}+\bar{p}_{s}\right)}\left(1-\frac{\mathcal{H}_{S}^{-1}}{\Delta \eta_{S}}\right) \frac{1}{\Delta \eta_{S^{\prime}}} \frac{2 \ell(\ell+1)}{\pi(2 \ell+1)^{2}} \\
& \times \int d k k^{4} P_{\sigma}(k) \dot{T}_{k}\left(\eta_{S}\right) \sum_{\tilde{\ell}=\ell-1, \ell+1} j_{\tilde{\ell}}\left(k \Delta \eta_{S}\right) \int_{\eta_{S^{\prime}}}^{\eta_{O}} d \eta\left(\eta_{O}-\eta\right)\left(\eta-\eta_{S^{\prime}}\right) \dot{T}_{k}(\eta) j_{\tilde{\ell}}(k \Delta \eta) \quad+\left(S \leftrightarrow S^{\prime}\right) \\
& \cong \frac{1}{8 \pi G a_{S}^{2}\left(\bar{\rho}_{S}+\bar{p}_{s}\right)}\left(1-\frac{\mathcal{H}_{S}^{-1}}{\Delta \eta_{S}}\right) \frac{1}{\Delta \eta_{S^{\prime}}} \frac{2 \ell(\ell+1)}{\pi(2 \ell+1)^{2}} \sum_{\tilde{\ell}=\ell-1, \ell+1}\left[\frac{I_{\tilde{\ell}}^{2} \tilde{\ell}^{2}}{\Delta \eta_{S}^{3}} P_{\sigma}\left(\frac{\tilde{\ell}}{\Delta \eta_{S}}\right) \dot{T}_{\tilde{\ell} / \Delta \eta_{S}}^{2}\left(\eta_{S}\right)\left(\eta_{S}-\eta_{S^{\prime}}\right) \Theta\left(\eta_{S}-\eta_{S^{\prime}}\right)\right] \\
& +\left(S \leftrightarrow S^{\prime}\right) .
\end{aligned}
$$

\section{Appendix D: Limber approximation}

In this work we have used the Limber approximation [25] repeatedly. It approximates the integral of the product of a spherical Bessel function and a slowly varying function (e.g., a power law) by

$$
\int_{x_{1}}^{x_{2}} d x f(x) j_{\ell}(x) \cong I_{\ell} f(\ell) \Theta\left(x_{2}-\ell\right) \Theta\left(\ell-x_{1}\right)
$$

for $x_{2}>x_{1}$, where $\Theta$ denotes the Heaviside function defined by

$$
\Theta(x)=\left\{\begin{array}{ll}
0, & x \leq 0 \\
1, & x>0
\end{array},\right.
$$

and $I_{\ell}^{2}=1.58 / \ell$ describes the area under the first peak of the spherical Bessel function $j_{\ell}(x)$. This rather crude approximation considers the contribution under the first peak only, and it usually gives an overestimation, but never by more than a factor of 2 [5] . Of course, if the function $f$ varies heavily in the region of the first peak, $\ell-1<x<\ell+1$, the approximation cannot be used.

We are, in particular, interested in (note that $\Delta \eta=$

$$
\left.\eta_{O}-\eta \text { and } \Delta \eta^{\prime}=\eta_{O}-\eta^{\prime}\right)
$$

$$
\begin{aligned}
& \int_{\eta_{S}}^{\eta_{O}} d \eta T(\eta) j_{\ell}(k \Delta \eta) \cong \frac{1}{k} T\left(\eta_{\ell, k}\right) I_{\ell} \Theta\left(\eta_{\ell, k}-\eta_{S}\right), \\
& \int_{\eta_{S}}^{\eta_{O}} d \eta T(\eta) j_{\ell}^{\prime}(k \Delta \eta) \\
& \cong \frac{1}{k} T\left(\eta_{\ell-1, k}\right) I_{\ell-1} \Theta\left(\eta_{\ell-1, k}-\eta_{S}\right) \\
& -\frac{1}{k} T\left(\eta_{\ell, k}\right) I_{\ell} \frac{\ell+1}{\ell} \Theta\left(\eta_{\ell, k}-\eta_{S}\right), \\
& \int_{\eta_{S}}^{\eta} d \eta^{\prime} \frac{\dot{T}\left(\eta^{\prime}\right)}{\left(k \Delta \eta^{\prime}\right)^{2}} j_{\ell}\left(k \Delta \eta^{\prime}\right) \\
& \cong \frac{1}{k \ell^{2}} \dot{T}\left(\eta_{\ell, k}\right) I_{\ell} \Theta\left(\eta_{\ell, k}-\eta_{S}\right) \Theta\left(\eta-\eta_{\ell, k}\right) . \\
& \int_{\eta_{S}}^{\eta_{O}} d \eta \int_{\eta_{S}}^{\eta} d \eta^{\prime} \frac{\dot{T}\left(\eta^{\prime}\right)}{\left(k \Delta \eta^{\prime}\right)^{2}} j_{\ell}\left(k \Delta \eta^{\prime}\right) \\
& \cong \frac{1}{k^{2} \ell} \dot{T}\left(\eta_{\ell, k}\right) I_{\ell} \Theta\left(\eta_{\ell, k}-\eta_{S}\right), \\
& \int_{\eta_{S}}^{\eta_{O}} d \eta\left(\eta-\eta_{S}\right)\left(\eta_{O}-\eta\right) \mathcal{H}(\eta) \dot{T}(\eta) \frac{j_{\ell}(k \Delta \eta)}{(k \Delta \eta)^{2}} \\
& \cong \frac{1}{\ell k^{2}}\left(\eta_{\ell, k}-\eta_{S}\right) I_{\ell} \mathcal{H}\left(\eta_{\ell, k}\right) \dot{T}\left(\eta_{\ell, k}\right) \Theta\left(\eta_{\ell, k}-\eta_{S}\right),
\end{aligned}
$$




$$
\begin{aligned}
& \int_{\eta_{S}}^{\eta_{O}} d \eta \int_{\eta_{S}}^{\eta} d \eta^{\prime} T\left(\eta^{\prime}\right) j_{\ell}^{\prime}\left(k \Delta \eta^{\prime}\right) \\
& \cong \frac{\ell-1}{k^{2}} T\left(\eta_{\ell-1, k}\right) I_{\ell-1} \Theta\left(\eta_{\ell-1, k}-\eta_{S}\right) \\
& \quad-\frac{\ell+1}{k^{2}} T\left(\eta_{\ell, k}\right) I_{\ell} \Theta\left(\eta_{\ell, k}-\eta_{S}\right), \\
& \int_{\eta_{S}}^{\eta_{O}} d \eta\left(\eta-\eta_{S}\right)\left(\eta_{O}-\eta\right) T_{k}(\eta) j_{\ell}(k \Delta \eta) \\
& \cong \frac{\ell}{k^{2}}\left(\eta_{\ell, k}-\eta_{S}\right) I_{\ell} T\left(\eta_{\ell, k}\right) \Theta\left(\eta_{\ell, k}-\eta_{S}\right), \\
& \int_{\eta_{S}}^{\eta_{O}} d \eta\left(\eta-\eta_{S}\right)\left(\eta_{O}-\eta\right) \dot{T}(\eta) j_{\ell}^{\prime}(k \Delta \eta)
\end{aligned}
$$

[1] H. Nussbaumer and L. Bieri, Discovering the Expanding Universe, Cambridge University Press (2009).

[2] B. Schmidt et al., Astrophys. J. 507, 46 (1998) arXiv:astro-ph/9805200.

A. Riess et al. Astron. J. 116, 1009 (1998) arXiv:astro-ph/9805201;

S. Perlmutter et al., Astrophys. J. 517, 565 (1999) arXiv:astro-ph/9812133;

See also http://www.nobelprize.org/nobel_prizes/ physics/laureates/2011/.

[3] R. Amanullah et al., Astrophys. J. 716, 712 (2010) arXiv:1004.1711;

J. Guy et al. (2010), arXiv:1010.4743v1;

T. Holsclaw, et al., Phys. Rev. Lett. 105, 241302 (2010) arXiv:1011.3079;

N. Suzuki et al. (2011) arXiv:1105.3470v1.

[4] L. Hui and P.B. Greene, Phys. Rev. D73, 123526 (2006) arXiv:astro-ph/0512159.

[5] C. Bonvin, R. Durrer and M.A. Gasparini, Phys. Rev. D73, 023523 (2006) arXiv:astro-ph/0511183.

[6] C. Bonvin, R. Durrer and M.A. Gasparini, Phys. Rev. D85, 029901(2012) arXiv:astro-ph/0511183.

[7] C. Bonvin, R. Durrer and M. Kunz, Phys. Rev. Lett. 96, 191302 (2006) arXiv:astro-ph/0603240.

[8] I. Ben-Dayan et al., JCAP $\mathbf{1 2 0 4}$ (2012) 036 arXiv:1202.1247.

[9] K. Enqvist, Gen Rel. Grav. 40, 451 (2008) arXiv:0709.2044, and references therein.

[10] E. Di Dio, M. Vonlanthen, and R. Durrer, JCAP 1202 (2012) 036 arXiv:1111.5764.

$$
\begin{aligned}
\cong & \frac{\ell-1}{k^{2}} I_{\ell-1}\left(\eta_{\ell-1, k}-\eta_{S}\right) \dot{T}\left(\eta_{\ell-1, k}\right) \Theta\left(\eta_{\ell-1, k}-\eta_{S}\right) \\
& -\frac{\ell+1}{k^{2}} I_{\ell}\left(\eta_{\ell, k}-\eta_{S}\right) \dot{T}\left(\eta_{\ell, k}\right) \Theta\left(\eta_{\ell, k}-\eta_{S}\right) .
\end{aligned}
$$

We have used the following propriety of the spherical Bessel functions 24]:

$$
j_{\ell}^{\prime}(k \Delta \eta)=j_{\ell-1}(k \Delta \eta)-\frac{\ell+1}{k \Delta \eta} j_{\ell}(k \Delta \eta) .
$$

[11] E. Di Dio, Master thesis at ETHZ (2010).

[12] T. Hui-Ching Lu, K. Ananda and C. Clarkson, Phys. Rev. D77, 043523 (2008) arXiv:0709.1619.

[13] K. N. Ananda, C. Clarkson and D. Wands, Phys. Rev. D75, 123518 (2007) arXiv:gr-qc/0612013 v1].

[14] E. Di Dio and R. Durrer, in preparation (2012).

[15] F. Schmidt and D. Jeong, arXiv:1204.3625

[16] D. Jeong, F. Schmidt, arXiv:1205.1512.

[17] P. Schneider, J. Ehlers and E.E. Falco, Gravitational Lenses, (Springer Verlag, 1992).

[18] G. F. R. Ellis, B. A. Bassett, P. K. Dunsby, Class. Quant. Grav. 15 (1998) 2345 arXiv:gr-qc/9801092.

[19] N. Straumann, General Relativity with Applications to Astrophysics, (Springer Verlag, Berlin, 2004).

[20] R. Durrer, Fund. Cos. Phys. 15, 209 (1994) arXiv:astro-ph/9311041; R. Durrer, The Cosmic Microwave Background, Cambridge University Press (2008).

[21] R. Durrer and T. Kahniashvili, Helv. Phys. Acta 71, 445 (1997) arXiv:astro-ph/9702226.

[22] D. Larsen et al., Astrophys.J.Suppl. 192, 16 (2011) [arXiv:1001:4635].

[23] V. Perlick, Living Reviews in Relativity, 7, 9 (2004).

[24] M. Abramowitz and I. Stegun, Handbook of Mathematical Functions, 9th Printing, Dover Publications, New York (1970).

[25] M. LoVerde and N. Afshordi, Phys. Rev. D78, 123506 (2008) arXiv:0809.5112. 